\title{
Integral Representations of the Catalan Numbers and Their Applications
}

\author{
Feng Qi ${ }^{1,2,3, *}$ and Bai-Ni Guo ${ }^{4}$ \\ 1 Institute of Mathematics, Henan Polytechnic University, Jiaozuo 454010, Henan Province, China \\ 2 College of Mathematics, Inner Mongolia University for Nationalities, Tongliao 028043, \\ Inner Mongolia Autonomous Region, China \\ 3 Department of Mathematics, College of Science, Tianjin Polytechnic University, Tianjin 300387, China \\ 4 School of Mathematics and Informatics, Henan Polytechnic University, Jiaozuo 454010, Henan Province, \\ China; bai.ni.guo@gmail.com \\ * Correspondence: qifeng618@gmail.com
}

Academic Editor: Hari M. Srivastava

Received: 22 May 2017; Accepted: 25 July 2017; Published: 3 August 2017

\begin{abstract}
In the paper, the authors survey integral representations of the Catalan numbers and the Catalan-Qi function, discuss equivalent relations between these integral representations, supply alternative and new proofs of several integral representations, collect applications of some integral representations, and present sums of several power series whose coefficients involve the Catalan numbers.
\end{abstract}

Keywords: Catalan number; integral representation; equivalent relation; application; sum of power series; Catalan-Qi function; Catalan-Qi number; beta function

\section{Introduction}

The Catalan numbers $C_{n}$ for $n \geq 0$ form a sequence of natural numbers that occur in various counting problems in combinatorial mathematics. The $n$th Catalan number can be expressed in terms of the central binomial coefficients $\left(\begin{array}{c}2 n \\ n\end{array}\right)$ by:

$$
C_{n}=\frac{1}{n+1}\left(\begin{array}{c}
2 n \\
n
\end{array}\right)=\frac{(2 n) !}{n !(n+1) !} .
$$

The Catalan numbers $C_{n}$ were described in the 18 th century by Leonhard Euler and are named after the Belgian mathematician Eugéne Charles Catalan. In 1988, it came to light that the Catalan numbers $C_{n}$ had been used in China by the Mongolian mathematician Ming Antu by 1730 [1-9]. In recent years, the Catalan numbers $C_{n}$ has been analytically generalized and studied in [10-26] and the closely related references therein. For more information on the Catalan numbers $C_{n}$, please refer to the monographs [27-30] and the closely related references therein.

\section{Integral Representations of the Catalan Numbers}

In this section, we recall integral representations of the Catalan numbers $C_{n}$ and their reciprocals $\frac{1}{C_{n}}$ and sketch their proofs as possible as we can.

\subsection{Penson-Sixdeniers' Integral Representations in 2001}

In 2001, Penson and Sixdeniers [31] established an integral representation by the Mellin transform. 
Theorem 1 ([31], p. 2, Equation (10)). For $n \geq 0$, the Catalan numbers $C_{n}$ can be represented by an integral:

$$
C_{n}=\frac{1}{2 \pi} \int_{0}^{4} \sqrt{\frac{4-x}{x}} x^{n} \mathrm{~d} x
$$

Proof. We rewrite the proof in [31] as follows. The Mellin transform of a real- or complex-valued function $f(x)$ is defined ([32], p. 29, Entry 1.14.32) by:

$$
\mathscr{M}(f ; s)=\int_{0}^{\infty} x^{s-1} f(x) \mathrm{d} x
$$

If $f(x)$ is continuous on $(0, \infty)$ and $\mathscr{M}(f ; \sigma+i t)$ is integrable on $(-\infty, \infty)$, then the inverse Mellin transform ([32], p. 29, Entry 1.14.35) reads that:

$$
f(x)=\frac{1}{2 \pi i} \int_{\sigma-i \infty}^{\sigma+i \infty} x^{-s} \mathscr{M}(f ; s) \mathrm{d} s
$$

Therefore, it is sufficient to compute the inverse Mellin transform:

$$
f(x)=\mathscr{M}^{-1}\left[\frac{4^{s-1} \Gamma\left(s-\frac{1}{2}\right)}{\sqrt{\pi} \Gamma(s+1)} ; x\right]=\frac{1}{2 \pi i} \int_{\sigma-i \infty}^{\sigma+i \infty} x^{-s} \frac{4^{s-1} \Gamma\left(s-\frac{1}{2}\right)}{\sqrt{\pi} \Gamma(s+1)} \mathrm{d} s,
$$

where the classical Euler gamma function $\Gamma(z)$ can be defined by:

$$
\Gamma(z)=\int_{0}^{\infty} t^{z-1} e^{-t} \mathrm{~d} t, \quad \Re(z)>0 .
$$

From the property:

$$
\mathscr{M}\left(x^{b} f\left(a x^{h}\right) ; s\right)=\frac{1}{h} a^{-(s+b) / h} \mathscr{M}\left(f\left(\frac{x+b}{h}\right) ; s\right)
$$

in [33], it follows immediately that:

$$
\mathscr{M}\left(\frac{1}{\sqrt{x}} f\left(\frac{x}{4}\right) ; s\right)=4^{s-1 / 2} \mathscr{M}\left(f\left(x-\frac{1}{2}\right) ; s\right) .
$$

Applying in Equation (4) the formula:

$$
\mathscr{M}\left[\left(1-x^{h}\right)_{+}^{\alpha-1} ; s\right]=\frac{1}{h} B\left(\alpha, \frac{s}{h}\right), \quad \Re(\alpha), s>0
$$

in [34] (p. 1102, Section 12.43, Entry 22) and [35] (p. 151, Entry 2.2(1)) to $h=1$ and $\alpha=\frac{3}{2}$ yields:

$$
f(x)=\frac{1}{\pi \sqrt{x}}\left(1-\frac{x}{4}\right)_{+}^{1 / 2}
$$

where:

$$
(y)_{+}^{\alpha-1}= \begin{cases}y^{\alpha-1}, & y>0 \\ 0, & y<0\end{cases}
$$

the classical beta function $B(z, w)$ can be defined by:

$$
B(z, w)=\int_{0}^{1} t^{z-1}(1-t)^{w-1} \mathrm{~d} t=\int_{0}^{\infty} \frac{t^{z-1}}{(1+t)^{z+w}} \mathrm{~d} t=\frac{\Gamma(z) \Gamma(w)}{\Gamma(z+w)}
$$


for $\Re(z), \Re(w)>0$. Then the desired integral representation of $C_{n}$ is proved.

Theorem 2 ([31], p. 3, Equation (16)). For $n \geq 0$, the sequence $n ! C_{n}$ can be represented by:

$$
n ! C_{n}=\frac{(2 n) !}{(n+1) !}=\int_{0}^{\infty}\left[\frac{1}{2} \operatorname{erf}\left(\frac{\sqrt{x}}{2}\right)+\frac{1}{\sqrt{\pi x}} e^{-x / 4}-\frac{1}{2}\right] x^{n} \mathrm{~d} x,
$$

where erf $(x)$ denotes the error function defined by:

$$
\operatorname{erf}(x)=\frac{2}{\sqrt{\pi}} \int_{0}^{x} e^{-t^{2}} \mathrm{~d} t
$$

Proof. We recite the proof in [31] as follows. This follows from applying the formula:

$$
\int_{0}^{\infty} x^{s-1}\left[\int_{0}^{\infty} h(y) f\left(\frac{x}{y}\right) \frac{\mathrm{d} y}{y}\right] \mathrm{d} x=\mathscr{M}(h ; s) \mathscr{M}(f ; s)
$$

in [32] (p. 29, Entries 1.14.39 and 1.14.40) to $h(x)=e^{-x}$ and the function $f(x)$ in (5).

By similar arguments, Penson and Sixdeniers [31] also derived:

$$
(n !)^{2} C_{n}=\frac{(2 n) !}{n+1}=\int_{0}^{\infty}\left[\frac{e^{\sqrt{x}}}{\sqrt{x}}+\operatorname{Ei}(-\sqrt{x})\right] x^{n} \mathrm{~d} x
$$

and an integral representation of the sequence $B_{n} C_{n}$, where $B_{n}$ is the Bell numbers [36-39] and $\mathrm{Ei}(y)$ is the exponential integral function which can be defined by:

$$
\operatorname{Ei}(y)=-\int_{-x}^{\infty} \frac{e^{-t}}{t} \mathrm{~d} t
$$

\subsection{Dana-Picard's Integral Representations in 2005}

In 2005, using a recurrence relation and the telescopic process, Dana-Picard [40] obtained integral representations for the Catalan numbers $C_{n}$ and their reciprocals $\frac{1}{C_{n}}$ respectively.

Theorem 3 ([40], Proposition 2.1 and Equation (9)). For $n \geq 0$, the Catalan numbers $C_{n}$ and their reciprocals $\frac{1}{C_{n}}$ can be represented by:

$$
C_{n}=\frac{1}{\pi} \int_{0}^{2} x^{2 n} \sqrt{4-x^{2}} \mathrm{~d} x
$$

and:

$$
\frac{1}{C_{n}}=\frac{(2 n+3)(2 n+2)(2 n+1)}{2^{4 n+4}} \int_{0}^{2} x^{2 n+1} \sqrt{4-x^{2}} \mathrm{~d} x .
$$

Proof. Now we sketch the proof in [40]. Let:

$$
I_{n}(a)=\int_{0}^{a} x^{n} \sqrt{a^{2}-x^{2}} \mathrm{~d} x, \quad n \geq 0 .
$$

Then $I_{0}=\frac{\pi}{4} a^{2}$ and:

$$
I_{n}(a)=a^{2} \frac{n-1}{n+2} I_{n-2}(a) .
$$

Using the telescopic method yields:

$$
I_{2 n}(a)=\pi\left(\frac{a}{2}\right)^{2 n+2} \frac{(2 n) !}{n !(n+1) !} \quad \text { and } \quad I_{2 n+1}(a)=a^{2 n+3} \frac{2^{2 n+1}}{(2 n+3)(2 n+2)(2 n+1)} \frac{n !(n+1) !}{(2 n !)} .
$$


Substituting (1) into the above equations and making use of Equation (11) result in:

$$
C_{n}=\frac{1}{\pi}\left(\frac{2}{a}\right)^{2 n+2} I_{2 n}(a)=\frac{1}{\pi}\left(\frac{2}{a}\right)^{2 n+2} \int_{0}^{a} x^{2 n} \sqrt{a^{2}-x^{2}} \mathrm{~d} x
$$

and:

$$
\begin{aligned}
\frac{1}{C_{n}} & =\frac{1}{a^{2 n+3}} \frac{(2 n+3)(2 n+2)(2 n+1)}{2^{2 n+1}} I_{2 n+1}(a) \\
& =\frac{1}{a^{2 n+3}} \frac{(2 n+3)(2 n+2)(2 n+1)}{2^{2 n+1}} \int_{0}^{a} x^{2 n+1} \sqrt{a^{2}-x^{2}} \mathrm{~d} x .
\end{aligned}
$$

Further setting $a=2$ leads to Equations (9) and (10) immediately.

\subsection{Dana-Picard's Integral Representations in 2010 and 2011}

In 2010, using separately three different substitutions, Dana-Picard [41] established the following integral representations for the Catalan numbers $C_{n}$ and their reciprocals $\frac{1}{C_{n}}$.

Theorem 4 ([41], Proposition 2.1). For $n \geq 0$, the Catalan numbers $C_{n}$ and their reciprocals $\frac{1}{C_{n}}$ can be represented by:

$$
C_{n}=\frac{2^{2 n+2}}{\pi} \int_{0}^{1} x^{2 n} \sqrt{1-x^{2}} \mathrm{~d} x
$$

and:

$$
\frac{1}{C_{n}}=\frac{(2 n+3)(2 n+2)(2 n+1)}{2^{2 n+1}} \int_{0}^{1} x^{2 n+1} \sqrt{1-x^{2}} \mathrm{~d} x .
$$

Proof. The sketch of the proof in [41] can be written as follows. For $n \geq 0$, let:

$$
A_{n}=\int_{0}^{1} x^{n} \sqrt{1-x^{2}} \mathrm{~d} x
$$

By the substitution $x=\sin u$ for $u \in\left[0, \frac{\pi}{2}\right]$, we can deduce:

$$
A_{n}=S_{n}-S_{n+2}
$$

where:

$$
S_{n}=\int_{0}^{\pi / 2} \sin ^{n} u \mathrm{~d} u
$$

Considering the well-known fact that:

$$
S_{2 p}=\frac{\pi}{2^{2 p+1}} \frac{(2 p) !}{(p !)^{2}}
$$

and using the Expression (1) derive:

$$
A_{2 p}=\frac{\pi}{2^{2 p+2}} \frac{(2 p) !}{p !(p+1) !}=\frac{\pi}{2^{2 p+2}} C_{p}
$$

and:

$$
A_{2 p+1}=\frac{2^{2 p}(p !)^{2}}{(2 p+3)(2 p+1) !}=\frac{2^{2 p+1}}{(2 p+3)(2 p+2)(2 p+1)} \frac{1}{C_{p}} .
$$

Accordingly, we acquire:

$$
C_{p}=\frac{2^{2 p+2}}{\pi} A_{2 p}=\frac{2^{2 p+2}}{\pi} \int_{0}^{1} x^{2 p} \sqrt{1-x^{2}} \mathrm{~d} x
$$


and:

$$
\begin{aligned}
\frac{1}{C_{p}} & =\frac{(2 p+3)(2 p+2)(2 p+1)}{2^{2 p+1}} A_{2 p+1} \\
& =\frac{(2 p+3)(2 p+2)(2 p+1)}{2^{2 p+1}} \int_{0}^{1} x^{2 p+1} \sqrt{1-x^{2}} \mathrm{~d} x .
\end{aligned}
$$

The integral representations (12) and (13) are thus proved.

Theorem 5 ([41], Proposition 3.1). For $n \geq 0$, the Catalan numbers $C_{n}$ can be represented by:

$$
C_{n}=\frac{2^{2 n+2}}{\pi} \int_{0}^{\infty} \frac{u^{2}}{\left(1+u^{2}\right)^{n+2}} \mathrm{~d} u .
$$

The outline of the proof in [41]. Using the substitution $u^{2}=\frac{1}{x^{2}}-1$ produces:

$$
A_{2 p}=\int_{0}^{\infty} \frac{u^{2}}{\left(1+u^{2}\right)^{p}} \mathrm{~d} u
$$

Combining this with Equation (14) yields (15).

The outline of the proof in [42]. It was stated in [43] that:

$$
\int_{0}^{\pi / 2} \sin ^{t} x \mathrm{~d} x=\frac{\sqrt{\pi}}{2} \frac{\Gamma\left(\frac{t+1}{2}\right)}{\Gamma\left(\frac{t+2}{2}\right)}, \quad t>-1
$$

See also ([44], p. 16, Equation (2.18)). Then it is not difficult to obtain:

$$
P_{n}=\int_{0}^{1} \frac{x^{n}}{\sqrt{1-x^{2}}} \mathrm{~d} x=\int_{0}^{\pi / 2} \sin ^{n} u \mathrm{~d} u= \begin{cases}\frac{\pi(2 p) !}{2^{2 p+1}(p !)^{2}}, & n=2 p \\ \frac{2^{2 p}(p !)^{2}}{(2 p+1) !}, & n=2 p+1\end{cases}
$$

On the other hand, using three irrational substitutions $u^{2}=\frac{1}{x^{2}}-1, u^{2}=1-x^{2}$, and $u=\sqrt{\frac{1-x}{1+x}}$ to compute $I_{n}$ produces:

$$
P_{n}=\int_{0}^{\infty}\left(1+u^{2}\right)^{-(n+2) / 2} \mathrm{~d} u=\int_{0}^{1}\left(1-u^{2}\right)^{(n-1) / 2} \mathrm{~d} u=2 \int_{0}^{1} \frac{\left(1-u^{2}\right)^{n}}{\left(1+u^{2}\right)^{n+1}} \mathrm{~d} u
$$

respectively. By similar argument to the proof of Theorem 4 and by the first formula in Equation (17), the integral representation (15) is verified once again.

A new proof the formula (15). In [34], p. 325, the fourth formula reads that:

$$
\int_{0}^{\infty} \frac{x^{\mu-1}}{\left(p+q x^{v}\right)^{n+1}} \mathrm{~d} x=\frac{1}{v p^{n+1}}\left(\frac{p}{q}\right)^{\mu / v} B\left(1+n-\frac{\mu}{v}, \frac{\mu}{v}\right)
$$

for $0<\frac{\mu}{v}<n+1$ and $p, q \neq 0$. Setting $p=q=1, \mu=3$, and $v=2$ and replacing $n$ by $n+1$ find:

$$
\int_{0}^{\infty} \frac{x^{2}}{\left(1+x^{2}\right)^{n+2}} \mathrm{~d} x=\frac{1}{2} B\left(\frac{2 n+1}{2}, \frac{3}{2}\right)=\frac{\pi}{2^{2 n+2}} C_{n}
$$

where we used in the last step the observation:

$$
C_{n}=\frac{1}{\pi} 2^{2 n+1} B\left(n+\frac{1}{2}, \frac{3}{2}\right)
$$


in [45] (Remark 6.2, Equation (6.1)). The formula (15) is thus proved.

Theorem 6 ([41], Proposition 4.1). For $n \geq 0$, the Catalan numbers $C_{n}$ can be represented by:

$$
C_{n}=\frac{2^{2 n+5}}{\pi} \int_{0}^{1} \frac{u^{2}\left(1-u^{2}\right)^{2 n}}{\left(1+u^{2}\right)^{2 n+3}} \mathrm{~d} u .
$$

The outline of the proof in [41]. Taking the substitution $u=\sqrt{\frac{1-x}{1+x}}$ concludes:

$$
A_{n}=8 \int_{0}^{1} \frac{u^{2}\left(1-u^{2}\right)^{2}}{\left(1+u^{2}\right)^{n+3}} \mathrm{~d} u .
$$

Combining this for even $n$ with Equation (14), we derive the integral presentation (19) immediately.

The outline of the proof in [42]. By same argument as in the proof of Theorem 4 and by the third formula in Equation (17), the integral representation (19) is verified once again.

\subsection{Dana-Picard-Zeitoun-Qi's Integral Representations in 2012 and 2016}

In 2012, Dana-Picard and Zeitoun [46] deduced an integral representation for the Catalan numbers $C_{n}$, which was corrected and developed by Qi [47] as the following integral representations.

Theorem 7 ([46], Corollary 3.2 and [47], Theorem 3.1). For $n \geq 0$ and $a>0$, the Catalan numbers $C_{n}$ can be represented by:

$$
\begin{aligned}
C_{n} & =\frac{1}{\pi} \frac{4^{n}}{n+1} \frac{1}{a^{2 n+1}} \int_{-a}^{a} x^{2 n} \sqrt{\frac{a+x}{a-x}} \mathrm{~d} x \\
& =\frac{1}{\pi} \frac{2^{2 n+1}}{n+1} \frac{1}{a^{2 n}} \int_{0}^{a} \frac{x^{2 n}}{\sqrt{a^{2}-x^{2}}} \mathrm{~d} x \\
& =\frac{1}{\pi} \frac{2^{2 n+1}}{n+1} \int_{0}^{\pi / 2} \sin ^{2 n} x \mathrm{~d} x
\end{aligned}
$$

and:

$$
\begin{aligned}
C_{n} & =\frac{1}{\pi} \frac{2^{2 n+1}}{2 n+1} \frac{1}{a^{2 n+2}} \int_{-a}^{a} x^{2 n+1} \sqrt{\frac{a+x}{a-x}} \mathrm{~d} x \\
& =\frac{1}{\pi} \frac{2^{2 n+2}}{2 n+1} \frac{1}{a^{2 n+2}} \int_{0}^{a} \frac{x^{2 n+2}}{\sqrt{a^{2}-x^{2}}} \mathrm{~d} x \\
& =\frac{1}{\pi} \frac{2^{2 n+2}}{2 n+1} \int_{0}^{\pi / 2} \sin ^{2 n+2} x \mathrm{~d} x .
\end{aligned}
$$

Proof. We sketch the proof in [47]. Let $a$ be a positive number. For $n \geq 0$, define:

$$
J_{n}=\int_{-a}^{a} x^{n} \sqrt{\frac{a+x}{a-x}} \mathrm{~d} x
$$

Then:

$$
J_{n}=\frac{1}{2} a^{n+1}\left(\left[1+(-1)^{n}\right] B\left(\frac{1}{2}, \frac{n+1}{2}\right)+\left[1+(-1)^{n+1}\right] B\left(\frac{1}{2}, \frac{n+2}{2}\right)\right)
$$

and:

$$
J_{n}=a^{n+1} \pi\left[\frac{1+(-1)^{n}}{n} \frac{1}{B\left(\frac{1}{2}, \frac{n}{2}\right)}+\frac{1+(-1)^{n+1}}{n+1} \frac{1}{B\left(\frac{1}{2}, \frac{n+1}{2}\right)}\right] .
$$

The Catalan numbers $C_{n}$ can be expressed in terms of the beta function $B(x, y)$ by:

$$
C_{n}=\frac{1}{\pi} \frac{4^{n}}{n+1} B\left(\frac{1}{2}, n+\frac{1}{2}\right) .
$$


Taking $n=2 p$ in Equation (22) and utilizing Equation (24) lead to:

$$
J_{2 p}=a^{2 p+1} B\left(\frac{1}{2}, \frac{2 p+1}{2}\right)=a^{2 p+1} \pi \frac{p+1}{4^{p}} C_{n}
$$

which is equivalent to:

$$
C_{n}=\frac{4^{n}}{n+1} \frac{1}{a^{2 n+1} \pi} J_{2 n}=\frac{1}{\pi} \frac{4^{n}}{n+1} \frac{1}{a^{2 n+1}} \int_{-a}^{a} x^{2 n} \sqrt{\frac{a+x}{a-x}} \mathrm{~d} x .
$$

The first Formula (20) thus follows.

By similar argument to the deduction of Equation (24), we can discover:

$$
C_{n}=\frac{4^{n+1}}{(2 n+1)(2 n+2)} \frac{1}{B\left(\frac{1}{2}, n+1\right)}, \quad n \geq 0
$$

Employing this identity and setting $n=2 p+1$ in Equation (23) figures out:

$$
J_{2 p+1}=a^{2 p+2} \frac{2 \pi}{2 p+2} \frac{1}{B\left(\frac{1}{2}, p+1\right)}=a^{2 p+2} \frac{2 \pi}{2 p+2} \frac{(2 p+1)(2 p+2)}{4^{p+1}} C_{p}
$$

which can be rearranged as:

$$
C_{p}=\frac{1}{a^{2 p+2}} \frac{1}{\pi} \frac{2^{2 p+1}}{2 p+1} J_{2 p+1}=\frac{1}{\pi} \frac{1}{a^{2 p+2}} \frac{2^{2 p+1}}{2 p+1} \int_{-a}^{a} x^{2 p+1} \sqrt{\frac{a+x}{a-x}} \mathrm{~d} x .
$$

The first formula in Equation (21) is thus proved.

The rest integral representations follow from mathematical techniques and changing variable of integration.

\subsection{Shi-Liu-Qi's Integral Representation in 2015}

In 2015, by virtue of an integral representation of the gamma function $\Gamma(x)$, Shi, Liu, and Qi [23] established an integral representation for the Catalan function:

$$
C_{x}=\frac{4^{x} \Gamma(x+1 / 2)}{\sqrt{\pi} \Gamma(x+2)}, \quad x>0 .
$$

Theorem 8 ([23], Theorem 1). For $x \geq 0$, the Catalan function $C_{x}$ can be represented by:

$$
C_{x}=\frac{e^{3 / 2} 4^{x}(x+1 / 2)^{x}}{\sqrt{\pi}(x+2)^{x+3 / 2}} \exp \left[\int_{0}^{\infty}\left(\frac{1}{e^{t}-1}-\frac{1}{t}+\frac{1}{2}\right) \frac{e^{-t / 2}-e^{-2 t}}{t} e^{-x t} \mathrm{~d} t\right] .
$$

Proof. This follows straightforwardly from applying the well-known formula:

$$
\ln \Gamma(z)=\ln \left(\sqrt{2 \pi} z^{z-1 / 2} e^{-z}\right)+\int_{0}^{\infty}\left(\frac{1}{e^{t}-1}-\frac{1}{t}+\frac{1}{2}\right) e^{-z t} \mathrm{~d} t, \quad \Re(z)>0
$$

in [48], Equation (3.22) to the logarithm of the Catalan function $C_{x}$.

\subsection{Qi-Shi-Liu's Integral Representations in 2015}

In 2015, by virtue of the Cauchy integral formula in the theory of complex functions, Qi and his two graduates, Shi and Liu, find an integral representation of the generating function $\frac{1}{1+\sqrt{1-4 x}}$ for the Catalan numbers $C_{n}$. Consequently, they derived an integral representation of the Catalan numbers $C_{n}$. 
Theorem 9 ([49], Theorem 1.4). The Catalan numbers $C_{n}$ for $n \geq 0$ can be represented by:

$$
C_{n}=\frac{1}{\pi} \int_{0}^{\infty} \frac{\sqrt{t}}{(t+1 / 4)^{n+2}} \mathrm{~d} t=\frac{2}{\pi} \int_{0}^{\infty} \frac{t^{2}}{\left(t^{2}+1 / 4\right)^{n+2}} \mathrm{~d} t .
$$

Proof. The Catalan numbers $C_{n}$ can be generated by:

$$
\frac{2}{1+\sqrt{1-4 x}}=\frac{1-\sqrt{1-4 x}}{2 x}=\sum_{n=0}^{\infty} C_{n} x^{n}
$$

By virtue of the Cauchy integral formula in the theory of complex functions, we discover:

$$
\frac{1}{1+\sqrt{1-4 x}}=\frac{1}{2 \pi} \int_{0}^{\infty} \frac{\sqrt{t}}{(t+1 / 4)(t-x+1 / 4)} \mathrm{d} t
$$

for $x \in\left(-\infty, \frac{1}{4}\right]$. Therefore, it follows that:

$$
\begin{aligned}
C_{n} & =\frac{1}{n !} \lim _{x \rightarrow 0} \frac{\mathrm{d}^{n}}{\mathrm{~d} x^{n}} \frac{2}{1+\sqrt{1-4 x}} \\
& =\frac{1}{\pi} \frac{1}{n !} \lim _{x \rightarrow 0} \frac{\mathrm{d}^{n}}{\mathrm{~d} x^{n}} \int_{0}^{\infty} \frac{\sqrt{t}}{(t+1 / 4)(t-x+1 / 4)} \mathrm{d} t \\
& =\frac{1}{\pi} \int_{0}^{\infty} \frac{\sqrt{t}}{(t+1 / 4)^{n+2}} \mathrm{~d} t .
\end{aligned}
$$

Further using the substitution $\sqrt{t}=s$ yields the second integral representation in Equation (26). The theorem is thus proved.

2.7. Qi's Integral Representations in 2017

In 2016, the first author discussed integral representations of the Catalan numbers $C_{n}$ once again. These results were formally published in 2017 and can be stated as the following theorem.

Theorem 10 ([45], Theorem 3.1 and Remark 6.6). The Catalan numbers $C_{n}$ for $n \geq 0$ can be represented by:

$$
C_{n}=\frac{2}{\pi(n+1)} \int_{0}^{2} \frac{x^{2 n}}{\sqrt{4-x^{2}}} \mathrm{~d} x=\frac{2^{2 n+1}}{\pi} \int_{0}^{1} \sqrt{\frac{1-t}{t}} t^{n} \mathrm{~d} t
$$

Proof. Using the substitution $x=a \sin s$ for $s \in\left[0, \frac{\pi}{2}\right]$ and employing Equation (16) for $t=n \geq 0$ reveal:

$$
I_{n}(a)=a^{n+2} \frac{\sqrt{\pi} \Gamma\left(\frac{n}{2}+\frac{1}{2}\right)}{4 \Gamma\left(\frac{n}{2}+2\right)}
$$

for $a>0$ and $n \geq 0$. Differentiating with respect to $a$ on both sides of Equation (11) gives:

$$
I_{n}^{\prime}(a)=a \int_{0}^{a} \frac{x^{n}}{\sqrt{a^{2}-x^{2}}} \mathrm{~d} x .
$$

On the other hand, differentiating with respect to $a$ on both sides of Equation (29) results in:

$$
I_{n}^{\prime}(a)=\frac{\sqrt{\pi}}{4}(n+2) a^{n+1} \frac{\Gamma\left(\frac{n}{2}+\frac{1}{2}\right)}{\Gamma\left(\frac{n}{2}+2\right)} .
$$


Combining Equation (30) with (31) and simplifying lead to:

$$
\int_{0}^{a} \frac{x^{n}}{\sqrt{a^{2}-x^{2}}} \mathrm{~d} x=\sqrt{\pi} a^{n} \frac{\Gamma\left(\frac{n}{2}+\frac{1}{2}\right)}{n \Gamma\left(\frac{n}{2}\right)}
$$

for $a>0$ and $n \geq 0$. The first representation in Equation (28) follows from combining:

$$
C_{n}=\frac{4^{n} \Gamma\left(n+\frac{1}{2}\right)}{\sqrt{\pi} \Gamma(n+2)}, \quad n \geq 0
$$

in [28] (p. 112, Equation (5.5)) with Equation (32).

The second integral representation in Equation (28) follows immediately from combining Equations (6) and (18). The desired proof is complete.

\subsection{Qi-Akkurt-Yildirim's Integral Representation}

In [11] (Theorem 1.1), an integral representation:

$$
C_{n}=\frac{k 2^{1+2 n(1-k)}}{\pi(n+1)} \int_{0}^{2} \frac{x^{(2 n+1) k-1}}{\sqrt{2^{2 k}-x^{2 k}}} \mathrm{~d} x
$$

for $k>0$ and $n \in \mathbb{N}$ was established.

\section{Catalan-Qi's Function and its Integral Representations}

In 2015, Qi and his coauthors generalized in [50] (Remark 1) and its formally published version [21] (Equation (9)) the Catalan numbers $C_{n}$ as the so-called Catalan-Qi function:

$$
C(a, b ; z)=\frac{\Gamma(b)}{\Gamma(a)}\left(\frac{b}{a}\right)^{z} \frac{\Gamma(z+a)}{\Gamma(z+b)}, \quad \Re(a), \Re(b)>0, \quad \Re(z) \geq 0 .
$$

It is clear that:

$$
C(b, a ; z)=\frac{1}{C(a, b ; z)} .
$$

When taking $x=n \in\{0\} \cup \mathbb{N}$, we call the quantities $C(a, b ; n)$ the Catalan-Qi numbers. It is easy to see that:

$$
C\left(\frac{1}{2}, 2 ; n\right)=C_{n} \quad \text { and } \quad C(a, b ; n)=\left(\frac{b}{a}\right)^{n} \frac{(a)_{n}}{(b)_{n}}
$$

for all $n \geq 0$, where:

$$
(x)_{n}=\prod_{\ell=0}^{n-1}(x+\ell)= \begin{cases}x(x+1) \cdots(x+n-1), & n \geq 1 \\ 1, & n=0\end{cases}
$$

is called the rising factorial or the Pochhammer symbol.

It is well known $[42,43,46,51,52]$ that the Wallis ratio is defined by:

$$
W_{n}=\frac{(2 n-1) ! !}{(2 n) ! !}=\frac{(2 n) !}{2^{2 n}(n !)^{2}}=\frac{1}{\sqrt{\pi}} \frac{\Gamma(n+1 / 2)}{\Gamma(n+1)}, \quad n \in \mathbb{N} .
$$

Hence, it is easy to see that:

$$
C_{n}=\frac{4^{n}}{n+1} W_{n}
$$

The Wallis ratio, or say, the ratio of two gamma functions, has been studied and applied by many mathematicians, see [44,51-57], for example, and plenty of literature therein. 
Now we are in a position to recall and to alternatively prove three integral representations of the Catalan-Qi function $C(a, b ; x)$ as follows.

Theorem 11 ([17], Equation (10)). For $b>a>0$ and $x \geq 0$, the Catalan-Qi function $C(a, b ; x)$ has the integral representation:

$$
C(a, b ; x)=\frac{1}{B(a, b-a)}\left(\frac{b}{a}\right)^{x} \int_{0}^{\infty}\left(1-e^{-u}\right)^{b-a-1} e^{-(x+a) u} \mathrm{~d} u .
$$

Proof. This follows from combination of the definition (35) and the integral formula:

$$
\frac{\Gamma(z+a)}{\Gamma(z+b)}=\frac{1}{\Gamma(b-a)} \int_{0}^{\infty}\left(1-e^{-u}\right)^{b-a-1} e^{-(z+a) u} \mathrm{~d} u, \quad b>a \geq 0
$$

in [48] (p. 67) for the ratio of two gamma functions $\Gamma(z+a)$ and $\Gamma(z+b)$.

Theorem 12 ([17], Theorem 4). For $b>a>0$ and $x \geq 0$, the Catalan-Qi function $C(a, b ; x)$ has integral representations:

$$
C(a, b ; x)=\left(\frac{a}{b}\right)^{b-1} \frac{1}{B(a, b-a)} \int_{0}^{b / a}\left(\frac{b}{a}-t\right)^{b-a-1} t^{x+a-1} \mathrm{~d} t
$$

and:

$$
C(a, b ; x)=\left(\frac{a}{b}\right)^{a} \frac{1}{B(a, b-a)} \int_{0}^{\infty} \frac{t^{b-a-1}}{(t+a / b)^{x+b}} \mathrm{~d} t .
$$

An alternative proof. Making use of the last formula in Equation (6) and the definition (35), we can rewritten the Catalan-Qi function $C(a, b ; x)$ as:

$$
C(a, b ; x)=\left(\frac{b}{a}\right)^{x} \frac{B(b, x+a)}{B(a, x+b)}
$$

and:

$$
C(a, b ; x)=\left(\frac{b}{a}\right)^{x} \frac{B(x+a, b-a)}{B(a, b-a)} .
$$

Applying Equation (6) into the factor $B(x+a, b-a)=B(b-a, x+a)$ in Equation (41) leads to:

$$
\begin{aligned}
C(a, b ; x) & =\left(\frac{b}{a}\right)^{x} \frac{1}{B(a, b-a)} \int_{0}^{1} t^{x+a-1}(1-t)^{b-a-1} \mathrm{~d} t \\
& =\left(\frac{b}{a}\right)^{x} \frac{1}{B(a, b-a)} \int_{0}^{b / a}\left(\frac{a}{b} s\right)^{x+a-1}\left[1-\left(\frac{a}{b} s\right)\right]^{b-a-1} \mathrm{~d}\left(\frac{a}{b} s\right) \\
& =\left(\frac{a}{b}\right)^{b-1} \frac{1}{B(a, b-a)} \int_{0}^{b / a}\left(\frac{b}{a}-s\right)^{b-a-1} s^{x+a-1} \mathrm{~d} s
\end{aligned}
$$

and:

$$
\begin{aligned}
C(a, b ; x) & =\left(\frac{b}{a}\right)^{x} \frac{1}{B(a, b-a)} \int_{0}^{\infty} \frac{t^{b-a-1}}{(1+t)^{x+b}} \mathrm{~d} t \\
& =\left(\frac{b}{a}\right)^{x} \frac{1}{B(a, b-a)} \int_{0}^{\infty} \frac{(b s / a)^{b-a-1}}{(1+b s / a)^{x+b}} \mathrm{~d}\left(\frac{b}{a} s\right) \\
& =\left(\frac{a}{b}\right)^{a} \frac{1}{B(a, b-a)} \int_{0}^{\infty} \frac{s^{b-a-1}}{(s+a / b)^{x+b}} \mathrm{~d} s
\end{aligned}
$$

respectively. The proof of Theorem 12 is thus complete. 


\section{Discussing Various Integral Representations}

In this section, we will discuss various integral representations recalled and proved above.

\subsection{Discussing Equation (2)}

Applying the substitution $x=4 t$ in Equation (2), rearranging, and employing the first definition in Equation (6) yield:

$$
C_{n}=\frac{1}{2 \pi} \int_{0}^{1} \sqrt{\frac{4-4 t}{4 t}}(4 t)^{n} \mathrm{~d}(4 t)=\frac{2^{2 n+1}}{\pi} \int_{0}^{1}(1-t)^{1 / 2} t^{n-1 / 2} \mathrm{~d} t=\frac{2^{2 n+1}}{\pi} B\left(\frac{3}{2}, n+\frac{1}{2}\right) .
$$

On the other hand, letting $a=\frac{1}{2}, b=2$, and $x=n \geq 0$ in Equation (41) and considering the first relation in Equation (37) give:

$$
C_{n}=4^{n} \frac{1}{B\left(\frac{1}{2}, \frac{3}{2}\right)} B\left(n+\frac{1}{2}, \frac{3}{2}\right)=\frac{2^{2 n+1}}{\pi} B\left(\frac{3}{2}, n+\frac{1}{2}\right) .
$$

As a result, the integral representation (2) is a special case of the integral representation (39). This can also be verified simpler by taking $a=\frac{1}{2}, b=2$, and $x=n \geq 0$ in Equation (39).

\subsection{Discussing Equation (7)}

By Equation (33) and $\Gamma(n+1)=n$ !, we obtain:

$$
n ! C_{n}=n ! \frac{4^{n} \Gamma\left(n+\frac{1}{2}\right)}{\sqrt{\pi}(n+1) !}=\frac{4^{n} \Gamma\left(n+\frac{1}{2}\right)}{\sqrt{\pi}(n+1)} .
$$

Combining this with Equation (7) and simplifying give:

$$
\begin{aligned}
\Gamma\left(n+\frac{1}{2}\right) & =\sqrt{\pi}(n+1) \int_{0}^{\infty}\left[\frac{1}{2} \operatorname{erf}\left(\frac{\sqrt{x}}{2}\right)+\frac{1}{\sqrt{\pi x}} e^{-x / 4}-\frac{1}{2}\right]\left(\frac{x}{4}\right)^{n} \mathrm{~d} x \\
& =2 \sqrt{\pi}(n+1) \int_{0}^{\infty}\left[\operatorname{erf}(\sqrt{t})+\frac{e^{-t}}{\sqrt{\pi t}}-1\right] t^{n} \mathrm{~d} t
\end{aligned}
$$

Hence, we guess that:

$$
\Gamma\left(x+\frac{1}{2}\right)=2 \sqrt{\pi}(x+1) \int_{0}^{\infty}\left[\operatorname{erf}(\sqrt{t})+\frac{e^{-t}}{\sqrt{\pi t}}-1\right] t^{x} \mathrm{~d} t, \quad x>-\frac{1}{2}
$$

which is equivalent to:

$$
\Gamma(x)=\sqrt{\pi}(2 x+1) \int_{0}^{\infty}\left[\operatorname{erf}(\sqrt{t})+\frac{e^{-t}}{\sqrt{\pi t}}-1\right] t^{x-1 / 2} \mathrm{~d} t, \quad x>0 .
$$

Actually, this can be derived from:

$$
\int_{0}^{\infty} \frac{e^{-t}}{\sqrt{\pi t}} t^{x-1 / 2} \mathrm{~d} t=\frac{1}{\sqrt{\pi}} \int_{0}^{\infty} e^{-t} t^{x-1} \mathrm{~d} t=\frac{\Gamma(x)}{\sqrt{\pi}}, \quad x>0,
$$


by the definition (3), and:

$$
\begin{gathered}
\int_{0}^{\infty}[\operatorname{erf}(\sqrt{t})-1] t^{x-1 / 2} \mathrm{~d} t=\frac{1}{x+1 / 2} \int_{0}^{\infty}[\operatorname{erf}(\sqrt{t})-1] \frac{\mathrm{d}}{\mathrm{d} t} t^{x+1 / 2} \mathrm{~d} t \\
=-\frac{1}{x+1 / 2} \int_{0}^{\infty}[\operatorname{erf}(\sqrt{t})-1]^{\prime} t^{x+1 / 2} \mathrm{~d} t=-\frac{1}{x+1 / 2} \int_{0}^{\infty} \frac{e^{-t}}{\sqrt{\pi} \sqrt{t}} t^{x+1 / 2} \mathrm{~d} t \\
=-\frac{2}{\sqrt{\pi}(2 x+1)} \int_{0}^{\infty} e^{-t} t^{x} \mathrm{~d} t=-\frac{2 \Gamma(x+1)}{\sqrt{\pi}(2 x+1)}, \quad x>-\frac{1}{2},
\end{gathered}
$$

by integration by part and the definition (8). In a word, we proved the integral representation (7) alternatively.

\subsection{Discussing Theorems 3 and 4}

By the substitution $x=2 t$, the integral representations (9) and (10) reduce to Equations (12) and (13). This can also be showed by letting $a=1$ in Equations (9) and (10). Consequently, the integral representations (9) and (10) are respectively equivalent to Equations (12) and (13).

By the substitution $x=\sqrt{t}$ in Equation (12) and by the first definition in Equation (6), we obtain:

$$
C_{n}=\frac{2^{2 n+2}}{\pi} \int_{0}^{1} t^{n} \sqrt{1-t} \frac{1}{2 \sqrt{t}} \mathrm{~d} t=\frac{2^{2 n+1}}{\pi} \int_{0}^{1} t^{n-1 / 2} \sqrt{1-t} \mathrm{~d} t=\frac{2^{2 n+1}}{\pi} B\left(n+\frac{1}{2}, \frac{3}{2}\right) .
$$

Accordingly, the integral representation (12) is a special case of the integral representation (39) and is equivalent to Equation (2).

Similarly, by the substitution $x=\sqrt{t}$ in Equation (13) and by the first definition in Equation (6), we acquire:

$$
\frac{1}{C_{n}}=\frac{(2 n+3)(2 n+2)(2 n+1)}{2^{2 n+2}} \int_{0}^{1} t^{n} \sqrt{1-t} \mathrm{~d} t=\frac{(2 n+3)(2 n+2)(2 n+1)}{2^{2 n+2}} B\left(n+1, \frac{3}{2}\right) .
$$

This implies that the integral representations (10) and (13) for reciprocals of the Catalan numbers $C_{n}$ can be alternatively verified by using Equations (33) and (6) in sequence as follows:

$$
\begin{aligned}
\frac{1}{C_{n}} & =\frac{\sqrt{\pi} \Gamma(n+2)}{4^{n} \Gamma\left(n+\frac{1}{2}\right)}=\frac{\sqrt{\pi}(n+1)\left(n+\frac{1}{2}\right)\left(n+\frac{3}{2}\right) \Gamma(n+1) \Gamma\left(\frac{3}{2}\right)}{4^{n} \Gamma\left(\frac{3}{2}\right) \Gamma\left(n+\frac{5}{2}\right)} \\
& =\frac{(2 n+3)(2 n+2)(2 n+1)}{2^{2 n+2}} B\left(n+1, \frac{3}{2}\right) \\
& =\frac{(2 n+3)(2 n+2)(2 n+1)}{2^{2 n+2}} \int_{0}^{1} t^{n} \sqrt{1-t} \mathrm{~d} t \\
& =\frac{(2 n+3)(2 n+2)(2 n+1)}{2^{2 n+1}} \int_{0}^{1} x^{2 n+1} \sqrt{1-x^{2}} \mathrm{~d} x \\
& =\frac{(2 n+3)(2 n+2)(2 n+1)}{2^{4 n+4}} \int_{0}^{2} x^{2 n+1} \sqrt{4-x^{2}} \mathrm{~d} x \\
& =\frac{(2 n+3)(2 n+2)(2 n+1)}{2^{2 n+1}} \frac{1}{a^{2 n+3}} \int_{0}^{a} x^{2 n+1} \sqrt{a^{2}-x^{2}} \mathrm{~d} x \\
& =\frac{(2 n+3)(2 n+2)(2 n+1)}{2^{2 n+2}} \frac{1}{a^{n+3 / 2}} \int_{0}^{a} t^{n} \sqrt{a-t} \mathrm{~d} t
\end{aligned}
$$

for $a>0$ and $n \geq 0$. 


\subsection{Discussing Equation (15)}

Using the substitution $u=\sqrt{t}$ in Equation (15) and considering the second expression (6) produce:

$$
\begin{aligned}
& \frac{2^{2 n+2}}{\pi} \int_{0}^{\infty} \frac{u^{2}}{\left(1+u^{2}\right)^{n+2}} \mathrm{~d} u=\frac{2^{2 n+2}}{\pi} \int_{0}^{\infty} \frac{t}{(1+t)^{n+2}} \frac{1}{2 \sqrt{t}} \mathrm{~d} t \\
& =\frac{2^{2 n+1}}{\pi} \int_{0}^{\infty} \frac{t^{1 / 2}}{(1+t)^{n+2}} \mathrm{~d} t=\frac{2^{2 n+1}}{\pi} B\left(\frac{3}{2}, n+\frac{1}{2}\right)=C_{n} .
\end{aligned}
$$

Hence, the integral representation (15) is proved once again.

\subsection{Discussing Equation (19)}

Letting $t=\frac{1-u^{2}}{1+u^{2}}$ in the integral of Equation (19) gives:

$$
\begin{gathered}
\int_{0}^{1} \frac{u^{2}\left(1-u^{2}\right)^{2 n}}{\left(1+u^{2}\right)^{2 n+3}} \mathrm{~d} u=\int_{0}^{1} \frac{1-t}{1+t}\left(\frac{1+t}{2}\right)^{3} t^{2 n} \frac{1}{(1+t)^{2}} \sqrt{\frac{1+t}{1-t}} \mathrm{~d} t \\
=\frac{1}{8} \int_{0}^{1} t^{2 n} \sqrt{1-t^{2}} \mathrm{~d} t=\frac{1}{16} \int_{0}^{1} s^{n-1 / 2} \sqrt{1-s} \mathrm{~d} s \\
=\frac{1}{16} B\left(\frac{3}{2}, n+\frac{1}{2}\right)=\frac{1}{16} \frac{\pi}{2^{2 n+1}} C_{n}=\frac{\pi}{2^{2 n+5}} C_{n} .
\end{gathered}
$$

The integral representation (19) is thus proved again.

\subsection{Discussing Equation (25)}

Currently we do not find any application of the integral representation (25) and do not derive any property of the Catalan numbers $C_{n}$ from the integral representation (25).

\subsection{Discussing Equation (26)}

By the substitution $t=\frac{u}{4}$ in the first integral of Equation (26) and comparing with the second integral in Equation (6) gives:

$$
\begin{gathered}
\int_{0}^{\infty} \frac{\sqrt{t}}{(t+1 / 4)^{n+2}} \mathrm{~d} t=\frac{1}{4} \int_{0}^{\infty} \frac{\sqrt{u / 4}}{(u / 4+1 / 4)^{n+2}} \mathrm{~d} u \\
=2^{2 n+1} \int_{0}^{\infty} \frac{\sqrt{u}}{(1+u)^{n+2}} \mathrm{~d} u=2^{2 n+1} B\left(\frac{3}{2}, n+\frac{1}{2}\right)=\pi C_{n} .
\end{gathered}
$$

Thus, the integral representations in Equation (26) are proved alternatively.

When changing the variable of integration by $t=\frac{u}{2}$ in the last representation in Equation (26), we can recover the integral representation (15).

\subsection{Discussing Equation (28)}

The first integral in Equation (28) can be computed as:

$$
\begin{gathered}
\int_{0}^{2} \frac{x^{2 n}}{\sqrt{4-x^{2}}} \mathrm{~d} x=\int_{0}^{2} \frac{(2 \sqrt{t})^{2 n}}{\sqrt{4-(2 \sqrt{t})^{2}}} \mathrm{~d}(2 \sqrt{t})=2^{2 n} \int_{0}^{1} \frac{t^{n}}{\sqrt{1-t}} \frac{1}{2 \sqrt{t}} \mathrm{~d} t \\
=2^{2 n-1} \int_{0}^{1} t^{n-1 / 2}(1-t)^{-1 / 2} \mathrm{~d} t=2^{2 n-1} B\left(n+\frac{1}{2}, \frac{1}{2}\right) .
\end{gathered}
$$


Then from Equation (24) it follows that:

$$
\int_{0}^{2} \frac{x^{2 n}}{\sqrt{4-x^{2}}} \mathrm{~d} x=2^{2 n-1} \pi \frac{n+1}{4^{n}} C_{n}
$$

which can be rewritten as Equation (28).

\subsection{Discussing Equation (34)}

The first integral representation (28) is a special case of the one Equation (34). Actually, the paper [11] was motivated by the article [45].

\subsection{Discussing Equation (38)}

By the substitution $e^{-u}=t$ in Equation (38) and by the first integral in Equation (6), we can see that the expressions (38) and (41) are equivalent to each other.

\subsection{Discussing Equations (39) and (40)}

When $a=\frac{1}{2}, b=2$, and $x=n \geq 0$, the integral representations (39) and (40) reduce to Equations (2) and (26) respectively.

Letting $a=\frac{1}{2}, b=2$, and $x=n \geq 0$ in Equation (41) results in the expression (18).

\subsection{The Beta Function and Reciprocals of the Catalan Numbers}

By Equation (33), the identity $\Gamma\left(\frac{1}{2}\right)=\sqrt{\pi}$, and the recurrence relation $\Gamma(x+1)=x \Gamma(x)$, it is easy to see that:

$$
\frac{1}{C_{n}}=\frac{\sqrt{\pi} \Gamma(n+2)}{4^{n} \Gamma\left(n+\frac{1}{2}\right)}=\frac{\left(n+\frac{1}{2}\right)(n+1)}{4^{n}} \frac{\Gamma\left(\frac{1}{2}\right) \Gamma(n+1)}{\Gamma\left(n+\frac{3}{2}\right)}=\frac{(2 n+1)(n+1)}{2^{2 n+1}} B\left(\frac{1}{2^{n}}, n+1\right)
$$

which is different from the one in Equation (42). Indeed, the Catalan numbers $C_{n}$ and their reciprocals $\frac{1}{C_{n}}$ can also be represented in terms of the beta functions $B\left(n+\ell-\frac{1}{2}, m+\frac{1}{2}\right)$ and $B\left(n+\ell, m+\frac{1}{2}\right)$ for $\ell, m \in \mathbb{N}$ respectively.

\section{Applications of Integral Representations}

Most of the above integral representations can be applied to discover properties of the Catalan numbers $C_{n}$. Now we recall some known applications of several integral representations of the Catalan numbers $C_{n}$.

\subsection{An Application of Equation (2)}

The integral representation (2) was applied in the proof of [22] (Theorem 5.1) to discover the identity:

$$
\sum_{\ell=0}^{\lfloor(j-1) / 2\rfloor}(-1)^{\ell}\left(\begin{array}{c}
j-\ell-1 \\
\ell
\end{array}\right) C_{i-\ell-1}=\frac{j}{i}\left(\begin{array}{c}
2 i-j-1 \\
i-1
\end{array}\right), \quad i \geq j \geq 1 .
$$

This identity generalizes:

$$
\sum_{k=0}^{\lfloor n / 2\rfloor}(-1)^{k}\left(\begin{array}{c}
n-k \\
k
\end{array}\right) C_{n-k}=1
$$

obtained in [58] (p. 2187, Theorem 2, Equation (15b)).

\subsection{An Application of Equation (19)}

The representation (19) was applied in [59] (p. 10) to compute several infinite series whose general terms involve binomial coefficients. 


\subsection{Applications of Equation (26)}

Recall from [60] (pp. 372-373) and [61] (p. 108, Definition 4) that a sequence $\left\{\mu_{n}\right\}_{0 \leq n \leq \infty}$ is said to be completely monotonic if its elements are non-negative and its successive differences are alternatively non-negative, that is:

$$
(-1)^{k} \Delta^{k} \mu_{n} \geq 0
$$

for $n, k \geq 0$, where:

$$
\Delta^{k} \mu_{n}=\sum_{m=0}^{k}(-1)^{m}\left(\begin{array}{c}
k \\
m
\end{array}\right) \mu_{n+k-m}
$$

Recall from [61] (p. 163, Definition 14a) that a completely monotonic sequence $\left\{a_{n}\right\}_{n \geq 0}$ is minimal if it ceases to be completely monotonic when $a_{0}$ is decreased.

Let $\lambda=\left(\lambda_{1}, \lambda_{2}, \ldots, \lambda_{n}\right) \in \mathbb{R}^{n}$ and $\mu=\left(\mu_{1}, \mu_{2}, \ldots, \mu_{n}\right) \in \mathbb{R}^{n}$. A sequence $\lambda$ is said to be majorized by $\mu$ (in symbols $\lambda \preceq \mu$ ) if:

$$
\sum_{\ell=1}^{k} \lambda_{[\ell]} \leq \sum_{\ell=1}^{k} \mu_{[\ell]}, \quad k=1,2, \ldots, n-1 \quad \text { and } \quad \sum_{\ell=1}^{n} \lambda_{\ell}=\sum_{\ell=1}^{n} \mu_{\ell}
$$

where $\lambda_{[1]} \geq \lambda_{[2]} \geq \cdots \geq \lambda_{[n]}$ and $\mu_{[1]} \geq \mu_{[2]} \geq \cdots \geq \mu_{[n]}$ are respectively the components of $\lambda$ and $\mu$ in decreasing order. A sequence $\lambda$ is said to be strictly majorized by $\mu$ (in symbols $\lambda \prec \mu$ ) if $\lambda$ is not a permutation of $\mu$. For example:

$$
\begin{aligned}
& (\underbrace{\frac{1}{n}, \ldots, \frac{1}{n}}_{n}) \prec(\underbrace{\frac{1}{n-1}, \ldots, \frac{1}{n-1}}_{n-1}, 0) \prec(\underbrace{\frac{1}{n-2}, \ldots, \frac{1}{n-2}}_{n-2}, 0,0) \prec \cdots \\
& \prec\left(\frac{1}{3}, \frac{1}{3}, \frac{1}{3}, 0, \ldots, 0\right) \prec\left(\frac{1}{2}, \frac{1}{2}, 0, \ldots, 0\right) \prec(1,0, \ldots, 0) .
\end{aligned}
$$

For more information on the theory of majorization and its applications, please refer to monographs $[62,63]$ and the closely related references therein.

Applying the integral representation (26), we can obtain properties and inequalities of the Catalan numbers $C_{n}$. Some of them can be recited as follows.

Theorem 13 ([49], Theorem 1.4). The sequence $\left\{\frac{C_{n}}{4^{n}}\right\}_{n \geq 0}$ is completely monotonic and minimal.

Theorem 14 ([49], Theorem 1.4). If $m \geq 1$ and $a_{0}, a_{1}, \ldots, a_{m}$ be non-negative integers, then:

$$
\left(\frac{C_{a_{0}}}{4^{a_{0}}}\right)^{m-1} \frac{C_{\sum_{k=0}^{m} a_{k}}}{4^{\sum_{k=0}^{m} a_{k}}} \geq \prod_{k=1}^{m} \frac{C_{a_{0}+a_{k}}}{4^{a_{0}+a_{k}}} \quad \text { and } \quad\left|\frac{C_{a_{i}+a_{j}}}{4^{a_{i}+a_{j}}}\right|_{m} \geq 0
$$

where $\left|e_{k j}\right|_{m}$ denotes a determinant of order $m$ with elements $e_{k j}$.

Theorem 15 ([49], Theorem 1.5). Let $m \in \mathbb{N}$ and let $n$ and $a_{k}$ for $1 \leq k \leq m$ be non-negative integers. Then the Catalan numbers $C_{n}$ satisfy:

$$
\left|(-1)^{a_{i}+a_{j}} \mathcal{C}_{n+a_{i}+a_{j}}\right|_{m} \geq 0 \text { and }\left|\mathcal{C}_{n+a_{i}+a_{j}}\right|_{m} \geq 0,
$$

where:

$$
\mathcal{C}_{\ell}=\ell ! C_{\ell}, \quad \ell \geq 0 .
$$


Theorem 16 ([49], Theorem 1.6). Let $m \in \mathbb{N}$ and let $\lambda$ and $\mu$ be two $m$-tuples of non-negative integers such that $\lambda \preceq \mu$. Then:

$$
\left|\prod_{i=1}^{m} \mathcal{C}_{n+\lambda_{i}}\right| \leq\left|\prod_{i=1}^{m} \mathcal{C}_{n+\mu_{i}}\right|
$$

where $\mathcal{C}_{\ell}$ is defined by (44). Consequently,

1. the infinite sequence $\left\{\mathcal{C}_{n}\right\}_{n \geq 0}$ is logarithmically convex,

2. the inequality $\mathcal{C}_{\ell+k}^{n} \leq \mathcal{C}_{\ell+n}^{k} \mathcal{C}_{\ell}^{n-k}$ is valid for $\ell \geq 0$ and $n>k>0$.

Theorem 17 ([49], Theorem 1.7). If $\ell \geq 0, n \geq k \geq m, k \geq n-k$, and $m \geq n-m$, then:

$$
\frac{C_{\ell+k} C_{\ell+n-k}}{C_{\ell+m} C_{\ell+n-m}} \geq \frac{(\ell+m) !(\ell+n-m) !}{(\ell+k) !(\ell+n-k) !} .
$$

For $n, m \in \mathbb{N}$ and $\ell \geq 0$, let:

$$
\begin{aligned}
\mathcal{G}_{n, m, \ell} & =\mathcal{C}_{\ell+n+2 m}\left(\mathcal{C}_{\ell}\right)^{2}-\mathcal{C}_{\ell+n+m} \mathcal{C}_{\ell+m} \mathcal{C}_{\ell}-\mathcal{C}_{\ell+n} \mathcal{C}_{\ell+2 m} \mathcal{C}_{\ell}+\mathcal{C}_{\ell+n}\left(\mathcal{C}_{\ell+m}\right)^{2}, \\
\mathcal{H}_{n, m, \ell} & =\mathcal{C}_{\ell+n+2 m}\left(\mathcal{C}_{\ell}\right)^{2}-2 \mathcal{C}_{\ell+n+m} \mathcal{C}_{\ell+m} \mathcal{C}_{\ell}+\mathcal{C}_{\ell+n}\left(\mathcal{C}_{\ell+m}\right)^{2}, \\
\mathcal{I}_{n, m, \ell} & =\mathcal{C}_{\ell+n+2 m}\left(\mathcal{C}_{\ell}\right)^{2}-2 \mathcal{C}_{\ell+n} \mathcal{C}_{\ell+2 m} \mathcal{C}_{\ell}+\mathcal{C}_{\ell+n}\left(\mathcal{C}_{\ell+m}\right)^{2}
\end{aligned}
$$

where $\mathcal{C}_{\ell}$ is defined by Equation (44). Then:

$$
\begin{gathered}
\mathcal{G}_{n, m, \ell} \geq 0, \quad \mathcal{H}_{n, m, \ell} \geq 0, \\
\mathcal{H}_{n, m, \ell} \lesseqgtr \mathcal{G}_{n, m, \ell} \quad \text { when } m \lessgtr n,
\end{gathered}
$$

and:

$$
\mathcal{I}_{n, m, \ell} \geq \mathcal{G}_{n, m, \ell} \geq 0 \quad \text { when } n \geq m \text {. }
$$

\subsection{Applications of Equation (39)}

Recall from [60] (Chapter XIII), [64] (Chapter 1) and [61] (Chapter IV) that an infinitely differentiable function $f$ is said to be completely monotonic on an interval $I$ if it satisfies $0 \leq(-1)^{k} f^{(k)}(x)<\infty$ on $I$ for all $k \geq 0$. It is known [61] (p. 161, Theorem 12b) that a function $f$ is completely monotonic on $(0, \infty)$ if and only if it is a Laplace transform $f(t)=\int_{0}^{\infty} e^{-t s} \mathrm{~d} \mu(s)$ of a positive measure $\mu$ defined on $[0, \infty)$ such that the above integral converges on $(0, \infty)$.

By virtue of the integral representation (39), we obtain asymptotic expansions and complete monotonicity related to the Catalan-Qi function.

Theorem 18 ([17], Theorem 4.2). For $b>a>0$, we have:

$$
C(a, b ; x)=\frac{1}{B(a, b-a)}\left(\frac{b}{a}\right)^{x} \sum_{k=0}^{\infty}(-1)^{k} \frac{\langle b-a-1\rangle_{k}}{k !} \frac{1}{x+a+k}
$$

where:

$$
\langle x\rangle_{n}=\prod_{k=0}^{n-1}(x-k)= \begin{cases}x(x-1) \cdots(x-n+1), & n \geq 1 \\ 1, & n=0\end{cases}
$$

is the falling factorial. Consequently, the function:

$$
(-1)^{\lfloor b-a\rfloor}\left[\left(\frac{a}{b}\right)^{x} C(a, b ; x)-\frac{1}{B(a, b-a)} \sum_{k=0}^{N}(-1)^{k} \frac{\langle b-a-1\rangle_{k}}{k !} \frac{1}{x+a+k}\right]
$$


for $N \in\{0\} \cup \mathbb{N}$ and $b>a>0$ is completely monotonic in $x \in[0, \infty)$, where $\lfloor x\rfloor$ denotes the floor function whose value is the largest integer less than or equal to $x$.

For more information and details on applications of the integral representations (26) and (39), please refer to [12-22] and the closely related references therein.

\section{Power Series whose Coefficients Involve Catalan Numbers}

In this section, we recall some results on sums of power series whose coefficients involve the Catalan numbers $C_{n}$ or the Catalan-Qi numbers $\left.C_{(} a, b ; n\right)$.

\subsection{Koshy-Gao's Result}

In 2012, Koshy and Gao [65] proved the following theorem.

Theorem 19 ([65]). For $|x|<4$, we have:

$$
\sum_{n=0}^{\infty} \frac{x^{n}}{C_{n}}= \begin{cases}1+\frac{x(4-x)^{3 / 2}+6 x(4-x)^{1 / 2}+24 \sqrt{x} \arcsin \frac{\sqrt{x}}{2}}{(4-x)^{5 / 2}}, & 0 \leq x<4 \\ 1-\frac{|x|(4-x)^{3 / 2}+6 \sqrt{|x|(4-x)}+24 \sqrt{|x|} \ln \frac{\sqrt{-x}+\sqrt{4-x}}{2}}{(4-x)^{5 / 2}}, & -4<x \leq 0 .\end{cases}
$$

Proof. We reformulate the proof by Koshy and Gao in [65] as follows. Denote:

$$
f(x)=\sum_{n=0}^{\infty} \frac{x^{n}}{C_{n}}
$$

Then:

$$
f^{\prime}(x)=\sum_{n=1}^{\infty} \frac{n x^{n-1}}{C_{n}}=\sum_{n=0}^{\infty} \frac{n+1}{C_{n+1}} x^{n} .
$$

Since $\frac{n+2}{C_{n}}=\frac{4 n+2}{C_{n+1}}$, by the recurrence relation, this yields:

$$
\begin{gathered}
\sum_{n=0}^{\infty} \frac{n+2}{C_{n}} x^{n}=\sum_{n=0}^{\infty} \frac{4 n+2}{C_{n+1}} x^{n}, \\
\sum_{n=0}^{\infty} \frac{n}{C_{n}} x^{n}+2 \sum_{n=0}^{\infty} \frac{x^{n}}{C_{n}}=\sum_{n=0}^{\infty} \frac{4(n+1)}{C_{n+1}} x^{n}-2 \sum_{n=0}^{\infty} \frac{x^{n}}{C_{n+1}}, \\
x f^{\prime}(x)+2 f(x)=4 f^{\prime}(x)-\frac{2}{x}[f(x)-1],
\end{gathered}
$$

and:

$$
x(x-4) f^{\prime}(x)+2(x+1) f(x)=2 .
$$

For $x \neq 0$, set $g(x)=\left|\frac{4-x}{x}\right|^{3 / 2}$. Then $\frac{g^{\prime}(x)}{g(x)}=-\frac{6}{x(4-x)}$. This implies that:

$$
[x(x-4) g(x)]^{\prime}=2(x+1) g(x) .
$$

Multiplying Equation (47) by $g(x)$, we obtain:

$$
x(x-4) f^{\prime}(x) g(x)+2(x+1) f(x) g(x)=2 g(x) .
$$

Using Equation (48), this can be rewritten as:

$$
[x(x-4) f(x) g(x)]^{\prime}=2 g(x) .
$$


Using Equation (48) again gives:

$$
\begin{gathered}
\{x(x-4)[f(x)-1] g(x)\}^{\prime}=[x(x-4) f(x) g(x)]^{\prime}-[x(x-4) g(x)]^{\prime} \\
=2 g(x)-2(x+1) g(x)=-2 x g(x) .
\end{gathered}
$$

Consequently:

$$
x(x-4)[f(x)-1] g(x)=-2 \int x g(x) \mathrm{d} x+\alpha_{1} \quad \text { and } \quad f(x)=1+\frac{2 \int x g(x) \mathrm{d} x-\alpha_{1}}{x(4-x) g(x)}
$$

where $\alpha_{1}$ is a constant.

For $0<x<4$, we have:

$$
\begin{aligned}
\int x g(x) \mathrm{d} x= & \int x\left(\frac{4-x}{x}\right)^{3 / 2} \mathrm{~d} x=\int \frac{(4-x)^{3 / 2}}{x^{1 / 2}} \mathrm{~d} x=2 \int\left(4-u^{2}\right)^{3 / 2} \mathrm{~d} u \quad\left(x=u^{2}\right) \\
& =\frac{1}{2} u\left(4-u^{2}\right)^{3 / 2}+3 u\left(4-u^{2}\right)^{1 / 2}+12 \arcsin \frac{u}{2}+\alpha_{2} \\
= & \frac{1}{2} \sqrt{x}(4-x)^{3 / 2}+3 \sqrt{x}(4-x)^{1 / 2}+12 \arcsin \frac{\sqrt{x}}{2}+\alpha_{2},
\end{aligned}
$$

where $\alpha_{2}$ is also a constant. Therefore, we have:

$$
\begin{aligned}
f(x) & =1+\frac{\sqrt{x}(4-x)^{3 / 2}+6 \sqrt{x}(4-x)^{1 / 2}+24 \arcsin \frac{\sqrt{x}}{2}+2 \alpha_{2}-\alpha_{1}}{x(4-x)\left(\frac{4-x}{x}\right)^{3 / 2}} \\
& =1+\frac{x(4-x)^{3 / 2}+6 x(4-x)^{1 / 2}+24 \sqrt{x} \arcsin \frac{\sqrt{x}}{2}+\alpha \sqrt{x}}{(4-x)^{5 / 2}}
\end{aligned}
$$

where $\alpha=2 \alpha_{2}-\alpha_{1}$. Since $f(0)=1=f^{\prime}(0)$, we have $\alpha=0$. Thus, the desired result for $0<x<4$ is proved.

For $-4<x<0$, by similar argument to the above, we acquire:

$$
\int x g(x) \mathrm{d} x=\frac{1}{2} \sqrt{|x|}(4-x)^{3 / 2}+3 \sqrt{|x|(4-x)}+12 \ln (\sqrt{|x|}+\sqrt{|4-x|})+\alpha_{3}
$$

and:

$$
f(x)=1-\frac{|x|(4-x)^{3 / 2}+6 \sqrt{|x|(4-x)}+24 \sqrt{|x|} \ln \frac{\sqrt{-x}+\sqrt{4-x}}{\alpha_{4}}}{(4-x)^{5 / 2}} .
$$

From $f(0)=1=f^{\prime}(0)$, we can determine $C_{4}=2$. The desired result is thus proved.

\subsection{Beckwith-Harbor-Abel's Result}

In 2014, Beckwith and Harbor [66] proposed a problem: show that:

$$
\sum_{n=0}^{\infty} \frac{2^{n}}{C_{n}}=5+\frac{3}{2} \pi \quad \text { and } \quad \sum_{n=0}^{\infty} \frac{3^{n}}{C_{n}}=22+8 \sqrt{3} \pi
$$

In 2016, Abel [67] answered this problem by proving a general result below.

Theorem 20 ([66,67]). For $0 \leq x<4$, we have:

$$
\sum_{n=0}^{\infty} \frac{x^{n}}{C_{n}}=1-\frac{x(x-10)}{(4-x)^{2}}+\frac{24 \sqrt{x}}{(4-x)^{5 / 2}} \arctan \sqrt{\frac{x}{4-x}} .
$$


Proof. We slightly modify the proof in [67] as follows. Using the beta integral:

$$
\int_{0}^{1} t^{m}(1-t)^{n} \mathrm{~d} t=\frac{m ! n !}{(m+n+1) !}
$$

gives:

$$
\begin{aligned}
\sum_{n=0}^{\infty} \frac{x^{n}}{C_{n}} & =1+\sum_{n=1}^{\infty} n(n+1) \frac{(n-1) ! n !}{(2 n) !} x^{n} \\
& =1+\sum_{n=1}^{\infty} n(n+1) x^{n} \int_{0}^{1} t^{n-1}(1-t)^{n} \mathrm{~d} t \\
& =1+\int_{0}^{1} \sum_{n=1}^{\infty} n(n+1) x^{n} t^{n-1}(1-t)^{n} \mathrm{~d} t
\end{aligned}
$$

for $|x|<4$. Further using:

$$
\sum_{n=1}^{\infty} n(n+1) z^{n}=\frac{2 z}{(1-z)^{3}}
$$

produces:

$$
\sum_{n=0}^{\infty} \frac{x^{n}}{C_{n}}=1+2 x \int_{0}^{1} \frac{1-t}{[1-x t(1-t)]^{3}} \mathrm{~d} t .
$$

Direct calculation of the integral yields the result (49).

\subsection{Lehmer's Result}

The editorial comment in [67] listed the formulas:

$$
\begin{aligned}
\sum_{n=0}^{\infty} \frac{1}{C_{n}} & =2+\frac{4 \pi}{9 \sqrt{3}}, & \sum_{n=0}^{\infty} \frac{(-1)^{n}}{C_{n}} & =\frac{14}{25}-\frac{24 \sqrt{5}}{125} \ln \frac{1+\sqrt{5}}{2}, \\
\sum_{n=0}^{\infty} \frac{(-2)^{n}}{C_{n}} & =\frac{1}{3}-\frac{1}{3 \sqrt{3}} \ln (2+\sqrt{3}), & \sum_{n=0}^{\infty} \frac{(-3)^{n}}{C_{n}} & =\frac{10}{49}-\frac{36}{49 \sqrt{21}} \ln \frac{5+\sqrt{21}}{2} .
\end{aligned}
$$

The editorial comment in [67] also pointed out that the result (45) had existed in [65], that the sum:

$$
\sum_{n=0}^{\infty} \frac{x^{n}}{C_{n}}=2 \frac{\sqrt{4-x}(8+x)+12 \sqrt{x} \arctan \frac{\sqrt{x}}{\sqrt{4-x}}}{\sqrt{(4-x)^{5}}}
$$

can be found on the website http://planetmath.org/, and that the problem by Beckwith and Harbor [66] can be solved easily from:

$$
\sum_{n=1}^{\infty} \frac{2^{n}}{\left(\begin{array}{c}
2 n \\
n
\end{array}\right)}=\frac{\pi}{2}+1, \quad \sum_{n=1}^{\infty} \frac{n 2^{n}}{\left(\begin{array}{c}
2 n \\
n
\end{array}\right)}=\pi+3, \quad \sum_{n=1}^{\infty} \frac{3^{n}}{\left(\begin{array}{c}
2 n \\
n
\end{array}\right)}=\frac{4 \pi \sqrt{3}}{3}+3, \quad \sum_{n=1}^{\infty} \frac{n 3^{n}}{\left(\begin{array}{c}
2 n \\
n
\end{array}\right)}=\frac{20 \pi \sqrt{3}}{3}+18
$$

which are special cases of the general formula in [68] (p. 452, Theorem) below.

Theorem 21 ([68], p. 452, Theorem). For $|x|<1$, we have:

$$
\frac{2 x \arcsin x}{\sqrt{1-x^{2}}}=\sum_{m=1}^{\infty} \frac{(2 x)^{2 m}}{m\left(\begin{array}{c}
2 m \\
m
\end{array}\right)} .
$$


Proof. Making use of the familiar Gregory series:

$$
t \arctan t=\sum_{m=1}^{\infty} \frac{(-1)^{m-1} t^{2 m}}{2 m-1}
$$

and setting $t=\frac{x}{\sqrt{1-x^{2}}}$ yields $\arctan t=\arcsin x$ and:

$$
\begin{aligned}
& \frac{x}{\sqrt{1-x^{2}}} \arcsin x=\sum_{m=1}^{\infty} \frac{(-1)^{m-1} x^{2 m}}{(2 m-1)\left(1-x^{2}\right)^{m}}=\sum_{m=1}^{\infty} \frac{(-1)^{m-1}}{2 m-1} \sum_{j=0}^{\infty}(-1)^{j}\left(\begin{array}{c}
-m \\
j
\end{array}\right) x^{2(j+m)} \\
& =\sum_{m=1}^{\infty} \frac{(-1)^{m-1}}{2 m-1} \sum_{j=0}^{\infty}\left(\begin{array}{c}
m+j-1 \\
j
\end{array}\right) x^{2(j+m)}=\sum_{r=1}^{\infty} x^{2 r} \sum_{m=1}^{r} \frac{(-1)^{m-1}(r-1) !}{(m-1) !(r-m) !(2 m-1)}
\end{aligned}
$$

Using Wallis' integral:

$$
\int_{0}^{\pi / 2}(\sin \theta)^{2 r-1} \mathrm{~d} \theta=\frac{2 \cdot 4 \cdot 6 \cdots(2 r-2)}{1 \cdot 3 \cdot 5 \cdots(2 r-1)}
$$

results in:

$$
\begin{gathered}
r\left(\begin{array}{c}
2 r \\
r
\end{array}\right) \sum_{v=0}^{r-1}(-1)^{v}\left(\begin{array}{c}
r-1 \\
v
\end{array}\right) \frac{1}{2 v+1}=r\left(\begin{array}{c}
2 r \\
r
\end{array}\right) \int_{0}^{1} \sum_{v=0}^{r-1}(-1)^{v}\left(\begin{array}{c}
r-1 \\
v
\end{array}\right) y^{2 v} \mathrm{~d} y \\
\quad=r\left(\begin{array}{c}
2 r \\
r
\end{array}\right) \int_{0}^{1}\left(1-y^{2}\right)^{r-1} \mathrm{~d} y=r\left(\begin{array}{c}
2 r \\
r
\end{array}\right) \int_{0}^{\pi / 2} \sin ^{2 r-1} \theta \mathrm{d} \theta=2^{2 r-1} .
\end{gathered}
$$

The sum (51) is thus proved.

From Equation (51), Lehmer [68] also derived:

$$
2(\arcsin x)^{2}=\sum_{m=1}^{\infty} \frac{(2 x)^{2 m}}{m^{2}\left(\begin{array}{c}
2 m \\
m
\end{array}\right)}, \quad \sum_{m=1}^{\infty} \frac{(2 x)^{2 m}}{m^{3}\left(\begin{array}{c}
2 m \\
m
\end{array}\right)}=4 \int_{0}^{x} \frac{(\arcsin y)^{2}}{y} \mathrm{~d} y
$$

and gave a recursive formula for:

$$
\sum_{m=1}^{\infty} \frac{m^{k-2}(2 x)^{2 m}}{\left(\begin{array}{c}
2 m \\
m
\end{array}\right)}
$$

Lehmer [68] (p. 454) pointed out that there are no known sum for interesting series of the form $\sum_{m=1}^{\infty} \frac{1}{m^{k}\left(\frac{2 m}{m}\right)}$ for $k \geq 5$.

\subsection{Beckwith-Harbor-Amdeberhan-Guan-Jiu-Moll-Vignat's Results}

In 2016, motivated by the above-mentioned problem posed by Beckwith and Harbor [66], Amdeberhan and his four coauthors [69] also proposed a general problem: find a closed-form formula for the series in Equation (46). They obtained the sum:

$$
\sum_{n=0}^{\infty} \frac{z^{n}}{C_{n}}={ }_{2} F_{1}\left(1,2 ; \frac{1}{2} ; \frac{z}{4}\right)=\frac{2(z+8)}{(4-z)^{2}}+\frac{24 \sqrt{z}}{(4-z)^{5 / 2}} \arcsin \frac{\sqrt{z}}{2}, \quad|z|<4
$$

by several methods, where ${ }_{2} F_{1}$ is the classical hypergeometric function which is a special case of the generalized hypergeometric series:

$$
{ }_{p} F_{q}\left(a_{1}, \ldots, a_{p} ; b_{1}, \ldots, b_{q} ; z\right)=\sum_{n=0}^{\infty} \frac{\left(a_{1}\right)_{n} \ldots\left(a_{p}\right)_{n}}{\left(b_{1}\right)_{n} \ldots\left(b_{q}\right)_{n}} \frac{z^{n}}{n !}
$$


defined for complex numbers $a_{i} \in \mathbb{C}$ and $b_{i} \in \mathbb{C} \backslash\{0,-1,-2, \ldots\}$, for positive integers $p, q \in \mathbb{N}$, and in terms of the rising factorial:

$$
(x)_{n}=\prod_{\ell=0}^{n-1}(x+\ell)= \begin{cases}x(x+1) \cdots(x+n-1), & n \geq 1 \\ 1, & n=0 .\end{cases}
$$

We observe that the Formulas (49) and (50) are the same one, that the sums (45) and (52) are the same one, and that, since:

$$
\arctan \sqrt{\frac{x}{4-x}}=\arcsin \frac{\sqrt{x}}{2}
$$

for $0 \leq x<4$, the four sums (49) to (52) are essentially the same one.

\section{Sums of Some New Series}

By applying some of the above-mentioned integral representations of the Catalan numbers $C_{n}$, we now construct some new finite and infinite power series.

\subsection{Sums of Two Finite and Infinite Series}

Making use of the integral representations (12) and (13), (15) and (19), (20) and (21), we can find the following finite and infinite power series involving the Catalan numbers $C_{n}$.

Theorem 22. For $k \geq 0$, we have the finite sums:

$$
\sum_{n=0}^{k} \frac{C_{n}}{2^{2 n}}=\frac{2}{\pi}\left[B\left(\frac{1}{2}, \frac{1}{2}\right)-B\left(\frac{1}{2}, k+\frac{3}{2}\right)\right]
$$

and:

$$
\sum_{n=0}^{k} \frac{2^{2 n}}{(n+1)(2 n+1)(2 n+3)} \frac{1}{C_{n}}=\frac{1}{2 \pi}\left[B\left(\frac{1}{2}, \frac{1}{2}\right)-B\left(\frac{1}{2}, k+\frac{3}{2}\right)\right] .
$$

Consequently, we have the infinite series:

$$
\sum_{n=0}^{\infty} \frac{C_{n}}{2^{2 n}}=2 \text { and } \sum_{n=0}^{\infty} \frac{2^{2 n}}{(n+1)(2 n+1)(2 n+3)} \frac{1}{C_{n}}=1 .
$$

Proof. Dividing the integral representations (12) and (13) and summing up over $0 \leq n \leq k$ give:

$$
\begin{gathered}
\sum_{n=0}^{k} \frac{C_{n}}{2^{2 n+2}}=\frac{1}{\pi} \int_{0}^{1}\left(\sum_{n=0}^{k} x^{2 n}\right) \sqrt{1-x^{2}} \mathrm{~d} x=\frac{1}{\pi} \int_{0}^{1} \frac{1-x^{2(k+1)}}{1-x^{2}} \sqrt{1-x^{2}} \mathrm{~d} x \\
=\frac{1}{\pi} \int_{0}^{1}\left(1-x^{2 k+2}\right)\left(1-x^{2}\right)^{-1 / 2} \mathrm{~d} x=\frac{1}{2 \pi} \int_{0}^{1}\left(t^{-1 / 2}-t^{k+1 / 2}\right)(1-t)^{-1 / 2} \mathrm{~d} t \\
=\frac{1}{2 \pi}\left[B\left(\frac{1}{2}, \frac{1}{2}\right)-B\left(k+\frac{3}{2}, \frac{1}{2}\right)\right] \rightarrow \frac{1}{2 \pi} B\left(\frac{1}{2}, \frac{1}{2}\right)=\frac{1}{2}
\end{gathered}
$$

and:

$$
\begin{gathered}
\sum_{n=0}^{k} \frac{2^{2 n+1}}{(2 n+3)(2 n+2)(2 n+1)} \frac{1}{C_{n}}=\int_{0}^{1}\left(\sum_{n=0}^{k} x^{2 n+1}\right) \sqrt{1-x^{2}} \mathrm{~d} x \\
=\int_{0}^{1} \frac{x\left(1-x^{2 k+2}\right)}{1-x^{2}} \sqrt{1-x^{2}} \mathrm{~d} x=\frac{1}{2} \int_{0}^{1}\left(1-t^{k+1}\right)(1-t)^{-1 / 2} \mathrm{~d} t \\
=\frac{1}{2}\left[B\left(\frac{1}{2}, 1\right)-B\left(\frac{1}{2}, k+2\right)\right] \rightarrow \frac{1}{2} B\left(\frac{1}{2}, 1\right)=1
\end{gathered}
$$


as $k \rightarrow \infty$.

Similarly, from Equations (15) and (19), it follows that:

$$
\begin{gathered}
\sum_{n=0}^{k} \frac{C_{n}}{2^{2 n+2}}=\frac{1}{\pi} \int_{0}^{\infty} \sum_{n=0}^{k} \frac{u^{2}}{\left(1+u^{2}\right)^{n+2}} \mathrm{~d} u=\frac{1}{\pi} \int_{0}^{\infty}\left[\frac{1}{1+u^{2}}-\frac{1}{\left(1+u^{2}\right)^{k+2}}\right] \mathrm{d} u \\
=\frac{1}{2}-\frac{1}{2 \pi} \int_{0}^{\infty} \frac{t^{-1 / 2}}{(1+t)^{k+2}} \mathrm{~d} t=\frac{1}{2}-\frac{1}{2 \pi} B\left(\frac{1}{2}, k+\frac{3}{2}\right) \rightarrow \frac{1}{2}
\end{gathered}
$$

and:

$$
\begin{gathered}
\sum_{n=0}^{k} \frac{C_{n}}{2^{2 n+5}}=\frac{1}{\pi} \int_{0}^{1} \frac{u^{2}}{\left(1+u^{2}\right)^{3}} \sum_{n=0}^{k}\left(\frac{1-u^{2}}{1+u^{2}}\right)^{2 n} \mathrm{~d} u=\frac{1}{\pi} \int_{0}^{1} \frac{u^{2}}{\left(1+u^{2}\right)^{3}} \frac{1-\left(\frac{1-u^{2}}{1+u^{2}}\right)^{2 k+2}}{1-\left(\frac{1-u^{2}}{1+u^{2}}\right)^{2}} \mathrm{~d} u \\
=\frac{1}{\pi} \int_{0}^{1} \frac{1-t}{1+t}\left(\frac{1+t}{2}\right)^{3} \frac{1-t^{2 k+2}}{1-t^{2}} \frac{1}{(1+t)^{2}} \sqrt{\frac{1+t}{1-t}} \mathrm{~d} t=\frac{1}{8 \pi} \int_{0}^{1} \frac{1-t^{2 k+2}}{\sqrt{1-t^{2}}} \mathrm{~d} t \\
=\frac{1}{16 \pi} \int_{0}^{1} \frac{1-s^{k+1}}{\sqrt{1-s}} \frac{1}{\sqrt{s}} \mathrm{~d} s=\frac{1}{16 \pi}\left[\int_{0}^{1}(1-s)^{-1 / 2} s^{-1 / 2} \mathrm{~d} s-\int_{0}^{1} s^{k+1 / 2}(1-s)^{-1 / 2} \mathrm{~d} s\right] \\
=\frac{1}{16 \pi}\left[B\left(\frac{1}{2}, \frac{1}{2}\right)-B\left(\frac{1}{2}, k+\frac{3}{2}\right)\right] \rightarrow \frac{1}{16}
\end{gathered}
$$

as $k \rightarrow \infty$. The proof of Theorem 22 is complete.

\subsection{Sums of Three Finite Series}

Applying the last integral expressions in Equations (20) and (21), we can obtain sums of three new finite series.

Theorem 23. For $k \geq 0$, we have:

$$
\sum_{n=0}^{k} \frac{n+1}{2^{2 n}} C_{n}=\frac{2}{B\left(\frac{1}{2}, k+1\right)}, \quad \sum_{n=0}^{k} \frac{2 n+1}{2^{2 n}} C_{n}=2\left[\frac{1}{B\left(\frac{1}{2}, k+2\right)}-1\right],
$$

and:

$$
\sum_{n=0}^{k} \frac{2^{2 n}}{(2 n+1)(n+1)} \frac{1}{C_{n}}=(k+1) B\left(\frac{1}{2}, k+1\right)-1 .
$$

When $k \rightarrow \infty$, these three series diverge.

Proof. Applying the last expressions in Equations (20) and (21) yields:

$$
\begin{aligned}
\sum_{n=0}^{k} \frac{n+1}{2^{2 n}} C_{n} & =\frac{2}{\pi} \int_{0}^{\pi / 2} \sum_{n=0}^{k} \sin ^{2 n} x \mathrm{~d} x=\frac{2}{\pi} \int_{0}^{\pi / 2} \frac{1-\sin ^{2 k+2} x}{\cos ^{2} x} \mathrm{~d} x \\
& =\frac{2}{\pi} \frac{\sqrt{\pi} \Gamma\left(k+\frac{3}{2}\right)}{\Gamma(k+1)}=\frac{2}{B\left(\frac{1}{2}, k+1\right)} \rightarrow \infty
\end{aligned}
$$

and:

$$
\begin{aligned}
& \sum_{n=0}^{k} \frac{2 n+1}{2^{2 n}} C_{n}=\frac{4}{\pi} \int_{0}^{\pi / 2} \sum_{n=0}^{k} \sin ^{2 n+2} x \mathrm{~d} x=\frac{4}{\pi} \int_{0}^{\pi / 2} \tan ^{2} x\left(1-\sin ^{2 k+2} x\right) \mathrm{d} x \\
& =\frac{4}{\pi}\left[\frac{\sqrt{\pi} \Gamma\left(k+\frac{5}{2}\right)}{\Gamma(k+2)}-\frac{\pi}{2}\right]=4\left[\frac{\Gamma\left(k+\frac{5}{2}\right)}{\sqrt{\pi} \Gamma(k+2)}-\frac{1}{2}\right]=2\left[\frac{1}{B\left(\frac{1}{2}, k+2\right)}-1\right] \rightarrow \infty
\end{aligned}
$$

as $k \rightarrow \infty$. 
From Equation (43), it follows that:

$$
\frac{2^{2 n+1}}{(2 n+1)(n+1)} \frac{1}{C_{n}}=B\left(\frac{1}{2}, n+1\right)=\int_{0}^{1}(1-t)^{-1 / 2} t^{n} \mathrm{~d} t .
$$

Summing up over $n$ from 0 to $k$ leads to:

$$
\begin{gathered}
\sum_{n=0}^{k} \frac{2^{2 n+1}}{(2 n+1)(n+1)} \frac{1}{C_{n}}=\int_{0}^{1}(1-t)^{-1 / 2} \sum_{n=0}^{k} t^{n} \mathrm{~d} t=\int_{0}^{1}(1-t)^{-1 / 2} \frac{1-t^{k+1}}{1-t} \mathrm{~d} t \\
=\int_{0}^{1}(1-t)^{-3 / 2}\left(1-t^{k+1}\right) \mathrm{d} t=2 \int_{0}^{1}\left(1-t^{k+1}\right)\left[(1-t)^{-1 / 2}\right]^{\prime} \mathrm{d} t \\
=-2+2(k+1) \int_{0}^{1} t^{k}(1-t)^{-1 / 2} \mathrm{~d} t=2\left[(k+1) B\left(\frac{1}{2}, k+1\right)-1\right] \rightarrow \infty
\end{gathered}
$$

as $k \rightarrow \infty$. The proof of Theorem 23 is complete.

\subsection{Sums of Three Infinite Power Series}

Now we use Equation (52) to derive sums of three infinite power series involving the reciprocal of the Catalan numbers $C_{n}$.

Theorem 24. The reciprocals $\frac{1}{C_{n}}$ of the Catalan numbers $C_{n}$ satisfy:

$$
\begin{gathered}
\sum_{n=0}^{\infty} \frac{z^{n}}{(n+1) C_{n}}=\sum_{n=0}^{\infty} \frac{z^{n}}{\left(\begin{array}{c}
2 n \\
n
\end{array}\right)}=\frac{4}{4-z}+\frac{4 \sqrt{z}}{(4-z)^{3 / 2}} \arcsin \frac{\sqrt{z}}{2}, \quad|z|<4, \\
\sum_{n=0}^{\infty} \frac{z^{n}}{(2 n+1) C_{n}}=\frac{2}{4-z}+\frac{8}{\sqrt{z}(4-z)^{3 / 2}} \arcsin \frac{\sqrt{z}}{2}, \quad|z|<4,
\end{gathered}
$$

and:

$$
\sum_{n=0}^{\infty} \frac{z^{n}}{(2 n+1)(n+1) C_{n}}=\frac{4}{\sqrt{z(4-z)}} \arcsin \frac{\sqrt{z}}{2}, \quad|z|<4
$$

Proof. Integrating on both sides of Equation (52) from 0 to $t$ with $|t|<4$ yields:

$$
\begin{aligned}
& \sum_{n=0}^{\infty} \frac{t^{n+1}}{(n+1) C_{n}}=\int_{0}^{t} \frac{24}{(4-z)^{2}} \mathrm{~d} z-\int_{0}^{t} \frac{2}{4-z} \mathrm{~d} z+\int_{0}^{t} \frac{24 \sqrt{z}}{(4-z)^{5 / 2}} \arcsin \frac{\sqrt{z}}{2} \mathrm{~d} z \\
= & \frac{6 t}{4-t}+2 \ln (4-t)-4 \ln 2+\int_{0}^{\arcsin \frac{\sqrt{t}}{2}} \frac{24 \sqrt{4 \sin ^{2} s}}{\left(4-4 \sin ^{2} s\right)^{5 / 2}} 8 \sin s \cos s \arcsin \frac{\sqrt{4 \sin ^{2} s}}{2} \mathrm{~d} s \\
= & \frac{6 t}{4-t}+2 \ln (4-t)-4 \ln 2+12 \int_{0}^{\arcsin \frac{\sqrt{t}}{2}} \frac{\sin ^{2} s}{\cos ^{4} s} s \mathrm{~d} s \\
= & \frac{6 t}{4-t}+2 \ln (4-t)-4 \ln 2+4 \int_{0}^{\arcsin \frac{\sqrt{t}}{2}} s\left(\tan ^{3} s\right)^{\prime} \mathrm{d} s \\
= & \frac{6 t}{4-t}+2 \ln (4-t)-4 \ln 2+4 \arcsin \frac{\sqrt{t}}{2} \tan ^{3} \arcsin \frac{\sqrt{t}}{2}-4 \int_{0}^{\arcsin \frac{\sqrt{t}}{2}} \tan ^{3} s \mathrm{~d} s \\
= & \frac{6 t}{4-t}+2 \ln (4-t)-4 \ln 2+\frac{4 t^{3 / 2}}{(4-t)^{3 / 2}} \arcsin _{\frac{\sqrt{t}}{2}}-4 \int_{0}^{\arcsin \frac{\sqrt{t}}{2}}\left(\tan s \sec ^{2} s-\tan s\right) \mathrm{d} s \\
= & \frac{6 t}{4-t}+2 \ln (4-t)-4 \ln 2+\frac{4 t^{3 / 2}}{(4-t)^{3 / 2}} \arcsin _{\frac{\sqrt{t}}{2}}-\frac{4 t}{8-2 t}+4\left[\ln 2-\frac{1}{2} \ln (4-t)\right] \\
= & \frac{4 t}{4-t}+\frac{4 t^{3 / 2}}{(4-t)^{3 / 2}} \arcsin \frac{\sqrt{t}}{2} .
\end{aligned}
$$


The Equality (54) is thus proved.

The Formula (52) can be rewritten as:

$$
\sum_{n=0}^{\infty} \frac{z^{2 n}}{C_{n}}=\frac{2\left(z^{2}+8\right)}{\left(4-z^{2}\right)^{2}}+\frac{24 z}{\left(4-z^{2}\right)^{5 / 2}} \arcsin \frac{z}{2}, \quad|z|<2 .
$$

Integrating on both sides of the above equality gives:

$$
\begin{aligned}
\sum_{n=0}^{\infty} \frac{t^{2 n+1}}{(2 n+1) C_{n}} & =\int_{0}^{t}\left[\frac{2\left(z^{2}+8\right)}{\left(4-z^{2}\right)^{2}}+\frac{24 z}{\left(4-z^{2}\right)^{5 / 2}} \arcsin \frac{z}{2}\right] \mathrm{d} z \\
& =\frac{3 t}{4-t^{2}}+\frac{1}{4} \ln \frac{2+t}{2-t}+3 \int_{0}^{\arcsin (t / 2)} \frac{\sin u}{\cos ^{4} u} u \mathrm{~d} u \\
& =\frac{3 t}{4-t^{2}}+\frac{1}{4} \ln \frac{2+t}{2-t}+\frac{8}{\left(4-t^{2}\right)^{3 / 2}} \arcsin \frac{t}{2}-\int_{0}^{\arcsin (t / 2)} \frac{1}{\cos ^{3} u} \mathrm{~d} u \\
& =\frac{3 t}{4-t^{2}}+\frac{1}{4} \ln \frac{2+t}{2-t}+\frac{8}{\left(4-t^{2}\right)^{3 / 2}} \arcsin \frac{t}{2}-\frac{t}{4-t^{2}}-\frac{1}{4} \ln \frac{2+t}{2-t} \\
& =\frac{2 t}{4-t^{2}}+\frac{8}{\left(4-t^{2}\right)^{3 / 2}} \arcsin \frac{t}{2}
\end{aligned}
$$

which can be rewritten as Equation (55).

Since $\frac{1}{(2 n+1)(n+1)}=\frac{2}{2 n+1}-\frac{1}{n+1}$, by Equations (54) and (55), we have:

$$
\begin{aligned}
& \sum_{n=0}^{\infty} \frac{x^{n}}{(2 n+1)(n+1) C_{n}}=\sum_{n=0}^{\infty}\left[\frac{2 x^{n}}{(2 n+1) C_{n}}-\frac{x^{n}}{(n+1) C_{n}}\right] \\
= & \sum_{n=0}^{\infty} \frac{2 x^{n}}{(2 n+1) C_{n}}-\sum_{n=0}^{\infty} \frac{x^{n}}{(n+1) C_{n}}=\frac{4}{\sqrt{z(4-z)}} \arcsin \frac{\sqrt{z}}{2} .
\end{aligned}
$$

The proof of Theorem 24 is complete.

\subsection{A New Proof for the Sum of a Power Series}

Now we supply a new proof for the following conclusion in [69] (pp. 115-116, Section 6).

Theorem 25 ([69], Section 6). For $x \geq 0$, we have:

$$
\sum_{n=0}^{\infty} \frac{1}{C_{n}} \frac{x^{n}}{n !}=1+\frac{1}{4} x+\frac{\sqrt{\pi}}{8}(x+6) \sqrt{x} e^{x / 4} \operatorname{erf}\left(\frac{\sqrt{x}}{2}\right) .
$$

Proof. In [21] (Theorem 1.5), it was obtained that:

$$
\sum_{n=0}^{\infty} C(a, b ; n) \frac{x^{n}}{n !}={ }_{1} F_{1}\left(a ; b ; \frac{b}{a} x\right) .
$$

Letting $a=2$ and $b=\frac{1}{2}$ in Equation (56) gives:

$$
\sum_{n=0}^{\infty} \frac{1}{C_{n}} \frac{x^{n}}{n !}={ }_{1} F_{1}\left(2 ; \frac{1}{2} ; \frac{x}{4}\right)
$$

Since:

$$
\operatorname{erf}(x)=\frac{2}{\sqrt{\pi}} e^{-x^{2}} \sum_{n=0}^{\infty} \frac{2^{k} z^{2 n+1}}{(2 n+1) ! !}
$$


see [34] (p. 889, 8.253) or [32] (p. 162, 7.6.2), it is straightforward to verify that:

$$
{ }_{1} F_{1}\left(2 ; \frac{1}{2} ; \frac{x}{4}\right)=1+\frac{1}{4} x+\frac{\sqrt{\pi}}{8}(x+6) \sqrt{x} e^{x / 4} \operatorname{erf}\left(\frac{\sqrt{x}}{2}\right) .
$$

The proof of Theorem 25 is thus complete.

7.5. More Sums of Series Involving Catalan or Catalan-Qi Numbers

Except [21] (Theorem 1.5), some series such as:

$$
\begin{gathered}
{ }_{2} F_{1}\left(a, 1 ; b ; \frac{b t}{a}\right)=\sum_{n=0}^{\infty} C(a, b ; n) t^{n}, \quad a, b>0 \\
\sum_{n=1}^{\infty}\left(\frac{a}{b}\right)^{n} C(a, b ; n)=\frac{a}{b-a-1}, \quad b>a+1>1 ;
\end{gathered}
$$

and:

$$
\sum_{n=0}^{\infty} C(a, b ; n) \frac{x^{2 n}}{(2 n) !}={ }_{1} F_{2}\left(a ; \frac{1}{2}, b ; \frac{b}{4 a} x^{2}\right), \quad a, b>0
$$

were also established in the papers [13] (Theorem 1) and [17] (Theorem 10).

In [24], among other things, it was obtained that:

$$
\sum_{n=0}^{\infty} \frac{(n+1)(2 n) ! ! C_{n}}{4^{n}(2 n+1)^{2}(2 n+1) ! !}=\frac{7}{8} \zeta(3)
$$

and:

$$
\sum_{n=0}^{\infty} \frac{x^{n}}{C_{n}}=\frac{24 \sqrt{-x}}{(4-x)^{5 / 2}} \ln \left(\frac{\sqrt{-x}+\sqrt{4-x}}{2}\right)+\frac{2 x}{(4-x)^{2}}+1, \quad x \in(-4,0],
$$

where $\zeta(z)$ denotes the Riemanian zeta function:

$$
\zeta(s)=\sum_{k=1}^{\infty} \frac{1}{k^{s}}, \quad \Re(s)>1 .
$$

\section{An Alternative Proof of the Formula (51)}

Substituting Equation (1) into the left-hand side of Equation (51) and making use of the identities in (36) and (37) give:

$$
\begin{gathered}
h\left(x^{2}\right)=\sum_{m=1}^{\infty} \frac{(2 x)^{2 m}}{m\left(\begin{array}{c}
2 m \\
m
\end{array}\right)}=\sum_{m=1}^{\infty} \frac{(2 x)^{2 m}}{m(m+1) C_{m}}=\sum_{m=1}^{\infty} \frac{(2 x)^{2 m}}{m(m+1) C\left(\frac{1}{2}, 2 ; m\right)} \\
=\sum_{m=1}^{\infty} \frac{(2 x)^{2 m}}{m(m+1)} C\left(2, \frac{1}{2}, ; m\right)=\sum_{m=1}^{\infty} \frac{(2 x)^{2 m}}{m(m+1)}\left(\frac{1}{4}\right)^{m} \frac{(2)_{m}}{\left(\frac{1}{2}\right)_{m}} \\
=\sum_{m=1}^{\infty} \frac{(2)_{m}}{\left(\frac{1}{2}\right)_{m}} \frac{x^{2 m}}{m(m+1)}=\sum_{m=1}^{\infty} \frac{(2)_{m}}{\left(\frac{1}{2}\right)_{m}} \frac{\left(x^{2}\right)^{m}}{m(m+1)} \\
=\sum_{m=1}^{\infty} \frac{(2)_{m}}{\left(\frac{1}{2}\right)_{m}} \frac{\left(x^{2}\right)^{m}}{m}-\frac{1}{x^{2}} \sum_{m=1}^{\infty} \frac{(2)_{m}}{\left(\frac{1}{2}\right)_{m}} \frac{\left(x^{2}\right)^{m+1}}{m+1} \triangleq h_{1}\left(x^{2}\right)-\frac{1}{x^{2}} h_{2}\left(x^{2}\right) .
\end{gathered}
$$


Differentiation and utilization of Equation (53) reveal:

$$
\begin{gathered}
h_{1}^{\prime}(t)=\sum_{m=1}^{\infty} \frac{(2)_{m}}{\left(\frac{1}{2}\right)_{m}} t^{m-1}=\frac{1}{t}\left[\sum_{m=0}^{\infty} \frac{(2)_{m}}{\left(\frac{1}{2}\right)_{m}} t^{m}-1\right]=\frac{1}{t}\left[\sum_{m=0}^{\infty} \frac{(2)_{m} m !}{\left(\frac{1}{2}\right)_{m}} \frac{t^{m}}{m !}-1\right] \\
=\frac{1}{t}\left[\sum_{m=0}^{\infty} \frac{(2)_{m}(1)_{m}}{\left(\frac{1}{2}\right)_{m}} \frac{t^{m}}{m !}-1\right]=\frac{1}{t}\left[2 F_{1}\left(1,2 ; \frac{1}{2} ; t\right)-1\right]
\end{gathered}
$$

and:

$$
h_{2}^{\prime}(t)=\sum_{m=1}^{\infty} \frac{(2)_{m}}{\left(\frac{1}{2}\right)_{m}} t^{m}=\sum_{m=1}^{\infty} \frac{(2)_{m}(1)_{m}}{\left(\frac{1}{2}\right)_{m}} \frac{t^{m}}{m !}={ }_{2} F_{1}\left(1,2 ; \frac{1}{2} ; t\right)-1
$$

Accordingly, we obtain:

$$
\begin{gathered}
h^{\prime}(t)=\left[h_{1}(t)-\frac{1}{t} h_{2}(t)\right]^{\prime}=h_{1}^{\prime}(t)-\frac{t h_{2}^{\prime}(t)-h_{2}(t)}{t^{2}}=\frac{1}{t}\left[{ }_{2} F_{1}\left(1,2 ; \frac{1}{2} ; t\right)-1\right]-\frac{h_{2}^{\prime}(t)}{t}+\frac{h_{2}(t)}{t^{2}} \\
=\frac{1}{t}\left[{ }_{2} F_{1}\left(1,2 ; \frac{1}{2} ; t\right)-1\right]-\frac{1}{t}\left[{ }_{2} F_{1}\left(1,2 ; \frac{1}{2} ; t\right)-1\right]+\frac{h_{2}(t)}{t^{2}}=\frac{h_{2}(t)}{t^{2}} .
\end{gathered}
$$

This implies that:

$$
\left[t^{2} h^{\prime}(t)\right]^{\prime}=h_{2}^{\prime}(t)={ }_{2} F_{1}\left(1,2 ; \frac{1}{2} ; t\right)-1 .
$$

Combining this with the right equality in Equation (52) leads to:

$$
\left[t^{2} h^{\prime}(t)\right]^{\prime}+1=\frac{2(4 t+8)}{(4-4 t)^{2}}+\frac{24 \sqrt{4 t}}{(4-4 t)^{5 / 2}} \arcsin \frac{\sqrt{4 t}}{2}=\frac{t+2}{2(1-t)^{2}}+\frac{3 \sqrt{t}}{2(1-t)^{5 / 2}} \arcsin \sqrt{t} .
$$

Integrating with respect to $t$ over $[0, x]$ for $0<x<1$ yields:

$$
\begin{aligned}
& x^{2} h^{\prime}(x)+x=\int_{0}^{x}\left[\frac{t+2}{2(1-t)^{2}}+\frac{3 \sqrt{t}}{2(1-t)^{5 / 2}} \arcsin \sqrt{t}\right] \mathrm{d} t \\
&=\frac{3 x}{2(1-x)}+\frac{1}{2} \ln (1-x)+3 \int_{0}^{\sqrt{x}} \frac{s^{2}}{\left(1-s^{2}\right)^{5 / 2}} \arcsin s \mathrm{~d} s \\
&=\frac{3 x}{2(1-x)}+\frac{1}{2} \ln (1-x)+3 \int_{0}^{\arcsin \sqrt{x}} \frac{u \sin ^{2} u \cos u}{\left(1-\sin ^{2} u\right)^{5 / 2}} \mathrm{~d} u \\
&=\frac{3 x}{2(1-x)}+\frac{1}{2} \ln (1-x)+3 \int_{0}^{\arcsin \sqrt{x}} \frac{u \sin ^{2} u}{\cos ^{4} u} \mathrm{~d} u \\
&=\frac{3 x}{2(1-x)}+\frac{1}{2} \ln (1-x)+\int_{0}^{\arcsin \sqrt{x}} u\left(\tan ^{3} u\right)^{\prime} \mathrm{d} u \\
&=\frac{3 x}{2(1-x)}+\frac{1}{2} \ln (1-x)+\arcsin \sqrt{x} \tan \arcsin \sqrt{x}-\int_{0}^{\arcsin \sqrt{x}} \tan ^{3} u \mathrm{~d} u \\
&=\frac{3 x}{2(1-x)}+\frac{1}{2} \ln (1-x)+\frac{x^{3 / 2}}{(1-x)^{3 / 2}} \arcsin \sqrt{x}-\int_{0}^{\arcsin \sqrt{x}}\left(\tan u \sec ^{2} u-\tan u\right) \mathrm{d} u \\
&=\frac{3 x}{2(1-x)}+\frac{1}{2} \ln (1-x)+\frac{x^{3 / 2}}{(1-x)^{3 / 2}} \arcsin \sqrt{x}-\frac{1}{2} \sec \arcsin ^{x}-\ln \cos \arcsin \sqrt{x}+\frac{1}{2} \\
&=\frac{2 x+1}{2(1-x)}+\frac{1}{2} \ln (1-x)+\frac{x^{3 / 2}}{(1-x)^{3 / 2}} \arcsin \sqrt{x}-\frac{1}{2(1-x)}-\frac{1}{2} \ln (1-x) \\
&=\frac{x}{1-x}+\frac{x^{3 / 2}}{(1-x)^{3 / 2}} \arcsin \sqrt{x} .
\end{aligned}
$$


Furthermore, similarly integrating gives:

$$
\begin{aligned}
h(t) & =\int_{0}^{t} \frac{1}{x^{2}}\left[\frac{x}{1-x}+\frac{x^{3 / 2}}{(1-x)^{3 / 2}} \arcsin \sqrt{x}-x\right] \mathrm{d} x \\
& =\int_{0}^{t}\left[\frac{1}{1-x}+\frac{1}{x^{1 / 2}(1-x)^{3 / 2}} \arcsin \sqrt{x}\right] \mathrm{d} x \\
& =-\ln (1-t)+\int_{0}^{\arcsin \sqrt{t}} \frac{2 s \sin s \cos s}{\left(1-\sin ^{2} s\right)^{3 / 2} \sin s} \mathrm{~d} s \\
& =-\ln (1-t)+2 \int_{0}^{\arcsin \sqrt{t}} \frac{s}{\cos ^{2} s} \mathrm{~d} s \\
& =-\ln (1-t)+2 \arcsin \sqrt{t} \tan \arcsin \sqrt{t}-2 \int_{0}^{\arcsin \sqrt{t}} \tan s \mathrm{~d} s \\
& =-\ln (1-t)+2 \sqrt{\frac{t}{1-t}} \arcsin \sqrt{t}+2 \ln \cos \arcsin \sqrt{t} \\
& =2 \sqrt{\frac{t}{1-t}} \arcsin \sqrt{t} .
\end{aligned}
$$

The proof of the Formula (51) is complete.

\section{Remarks}

Finally, we list several remarks on closely related results.

Remark 1. It seems that there are close and similar ideas in [66,69] and that the paper [69] is almost an expanded version of [66]. Great minds think alike!

Remark 2. In [68] (p. 452, Theorem), it was established that:

$$
\sum_{m=1}^{\infty} \frac{(2 x)^{2 m}}{m\left(\begin{array}{c}
2 m \\
m
\end{array}\right)}=\frac{2 x \arcsin x}{\sqrt{1-x^{2}}}, \quad|x|<1 .
$$

This can be rearranged as:

$$
\sum_{m=1}^{\infty} \frac{(2 x)^{2 m}}{m(m+1) C_{m}}=\frac{2 x \arcsin x}{\sqrt{1-x^{2}}}, \quad|x|<1 .
$$

Remark 3. Letting $a=\frac{1}{2}$ and $b=2$ in Equation (57) and comparing with Equation (27) leads to:

$$
{ }_{2} F_{1}\left(\frac{1}{2}, 1 ; 2 ; 4 x\right)=\frac{1-\sqrt{1-4 x}}{2 x}, \quad|x| \leq \frac{1}{4} .
$$

This can also be deduced from the formula:

$$
{ }_{2} F_{1}(a, b ; c ; z)=\frac{1}{B(b, c-b)} \int_{0}^{1} \frac{t^{b-1}(1-t)^{c-b-1}}{(1-t z)^{a}} \mathrm{~d} t, \quad \Re(c)>\Re(b)>0
$$

in [70] (p. 558, 15.3.1) and [34] (9.111).

Remark 4. Letting $a=\frac{1}{2}$ and $b=2$ in Equation (58) gives:

$$
\sum_{n=1}^{\infty} \frac{C_{n}}{4^{n}}=1
$$


which can be rewritten as:

$$
\sum_{n=0}^{\infty} \frac{(2 n+1) ! !}{(2 n+4) ! !}=\frac{1}{2}
$$

Remark 5. Taking $a=2$ and $b=\frac{1}{2}$ in Equation (59) results in:

$$
\sum_{n=0}^{\infty} \frac{1}{C_{n}} \frac{x^{2 n}}{(2 n) !}={ }_{1} F_{2}\left(2 ; \frac{1}{2}, \frac{1}{2} ; \frac{x^{2}}{16}\right)=1+\frac{\pi}{16} x\left[x L_{-1}\left(\frac{x}{2}\right)+6 L_{0}\left(\frac{x}{2}\right)\right]
$$

where:

$$
\mathbf{L}_{v}=\left(\frac{z}{2}\right)^{v+1} \sum_{n=0}^{\infty} \frac{1}{\Gamma\left(n+\frac{3}{2}\right) \Gamma\left(n+v+\frac{3}{2}\right)}\left(\frac{z}{2}\right)^{2 n}
$$

denotes the modified Struve function, see [32] ( $p$. 228, 11.2.2).

Remark 6. This paper is a slightly modified version of the preprint [71].

Acknowledgments: The authors are thankful to Wei-Dong Jiang (Weihai Vocational University, China), to Chun-Na Zeng (Chongqing Normal University, China), and to Victor H. Moll (Tulane University, USA) for their kind helps in October 2016 to find and supply the formally published versions of the papers [67,69].

Author Contributions: The authors contributed equally to this work.

Conflicts of Interest: The authors declare no conflict of interest.

\section{References}

1. Larcombe, P. On the history of the Catalan numbers: A first record in China. Math. Today (Southend-on-Sea) 1999, 35, 89.

2. Larcombe, P.J. The 18th century Chinese discovery of the Catalan numbers. Math. Spectr. 1999/2000, 32, 5-7.

3. Liu, J.J. Ming Antu and Catalan numbers. J. Math. Res. Expos. 2002, 22, 589-594. (In Chinese)

4. Luo, J.J. Ming Antu, the first discoverer of the Catalan numbers. Neimenggu Daxue Xuebao 1988, 19, $239-245$. (In Chinese)

5. Luo, J.J. Catalan numbers in the history of mathematics in China. In Combinatorics and Graph Theory (Hefei, 1992); World Scientific Publishing: River Edge, NJ, USA, 1993; pp. 68-70.

6. Luo, J.J. Ming Antu and his power series expansions. In Seki, Founder of Modern Mathematics in Japan; Springer Proceedings in Mathematics and Statistics; Springer: Tokyo, Japan, 2013; Volume 39, pp. 299-310. doi:10.1007/978-4-431-54273-5_20.

7. Ma, X.R. Notes on a result due to Ming Antu. J. Math. Res. Expos. 2002, 22, 595-598. (In Chinese)

8. Ma, X.R. The general solution of Ming Antu's problem. Acta Math. Sin. (Engl. Ser.) 2004, 20, 157-162. doi:10.1007/s10114-003-0282-2 .

9. Te, G.S. Catalan numbers in the Xiang shu yi yuan. In Collected Research Papers on the History of Mathematics; Inner Mongolia University Press: Hohhot, China, 1991; Volume 2, pp. 105-112. (In Chinese)

10. Liu, F.-F.; Shi, X.-T.; Qi, F. A logarithmically completely monotonic function involving the gamma function and originating from the Catalan numbers and function. Glob. J. Math. Anal. 2015, 3, 140-144. doi:10.14419/gjma.v3i4.5187.

11. Qi, F.; Akkurt, A.; Yildirim, H. Catalan numbers, $k$-gamma and $k$-beta functions, and parametric integrals. J. Comput. Anal. Appl. 2018, 25, 1036-1042.

12. Qi, F.; Cerone, P. Several Expressions, Some Properties, and a Double Inequality of the Fuss-Catalan Numbers; ResearchGate Research: Berlin, Germany, 2015. doi:10.13140/RG.2.1.1655.6004

13. Mahmoud, M.; Qi, F. Three identities of the Catalan-Qi numbers. Mathematics 2016, 4, 35. doi:10.3390/math4020035.

14. Qi, F.; Guo, B.-N. Logarithmically complete monotonicity of a function related to the Catalan-Qi function. Acta Univ. Sapientiae Math. 2016, 8, 93-102. doi:10.1515/ausm-2016-0006.

15. Qi, F.; Guo, B.-N. Logarithmically complete monotonicity of Catalan-Qi function related to Catalan numbers. Cogent Math. 2016, 3, 1179379. doi:10.1080/23311835.2016.1179379. 
16. Qi, F.; Guo, B.-N. Some properties and generalizations of the Catalan, Fuss, and Fuss-Catalan numbers. In Mathematical Analysis and Applications: Selected Topics; Dutta, H., Ruzhansky, M., Agarwal, R.P., Eds.; Wiley: Hoboken, NJ, USA; ResearchGate Research: Berlin, Germany, 2015. doi:10.13140/RG.2.1.1778.3128.

17. Qi, F.; Mahmoud, M.; Shi, X.-T.; Liu, F.-F. Some properties of the Catalan-Qi function related to the Catalan numbers. SpringerPlus 2016, 5, 1126. doi:10.1186/s40064-016-2793-1.

18. Qi, F.; Shi, X.-T.; Cerone, P. A Unified Generalization of the Catalan, Fuss, and Fuss-Catalan Numbers and the Catalan-Qi Function; ResearchGate Working Paper; ResearchGate Research: Berlin, Germany, 2015. doi:10.13140/RG.2.1.3198.6000.

19. Qi, F.; Shi, X.-T.; Liu, F.-F.; Kruchinin, D.V. Several formulas for special values of the Bell polynomials of the second kind and applications. J. Appl. Anal. Comput. 2017, 7, 857-871. doi:10.11948/2017054.

20. Qi, F.; Shi, X.-T.; Mahmoud, M.; Liu, F.-F. Schur-convexity of the Catalan-Qi function related to the Catalan numbers. Tbilisi Math. J. 2016, 9, 141-150. doi:10.1515/tmj-2016-0026.

21. Qi, F.; Shi, X.-T.; Mahmoud, M.; Liu, F.-F. The Catalan numbers: a generalization, an exponential representation, and some properties. J. Comput. Anal. Appl. 2017, 23, 937-944.

22. Qi, F.; Zou, Q.; Guo, B.-N. Identities of the Chebyshev Polynomials, the Inverse of a Triangular Matrix, and Identities of the Catalan Numbers; ResearchGate Working Paper; ResearchGate Research: Berlin, Germany, 2017. doi:10.13140/RG.2.2.27344.71684.

23. Shi, X.-T.; Liu, F.-F.; Qi, F. An integral representation of the Catalan numbers. Glob. J. Math. Anal. 2015, 3, 130-133. doi:10.14419/gjma.v3i3.5055.

24. Yin, L.; Qi, F. Several series identities involving the Catalan numbers. Preprints 2017, 2017030029. doi:10.20944/preprints201703.0029.v1.

25. Zou, Q. Analogues of several identities and supercongruences for the Catalan-Qi numbers. J. Inequal. Spec. Funct. 2016, 7, 235-241.

26. Zou, Q. The $q$-binomial inverse formula and a recurrence relation for the $q$-Catalan-Qi numbers. J. Math. Anal. 2017, 8, 176-182.

27. Grimaldi, R.P. Fibonacci and Catalan Numbers: An Introduction; John Wiley \& Sons, Inc.: Hoboken, NJ, USA, 2012. doi:10.1002/9781118159743.

28. Koshy, T. Catalan Numbers with Applications; Oxford University Press: Oxford, UK, 2009.

29. Roman, S. An Introduction to Catalan Numbers, with a foreword by Richard Stanley; Compact Textbook in Mathematics; Birkhäuser/Springer: Cham, Switzerland, 2015. doi:10.1007/978-3-319-22144-1.

30. Stanley, R.P. Catalan Numbers; Cambridge University Press: New York, NY, USA, 2015. doi:10.1017/CBO9781139871495.

31. Penson, K.A.; Sixdeniers, J.-M. Integral representations of Catalan and related numbers. J. Integer Seq. 2001, 4, Art. 01.2.5.

32. Olver, F.W.J.; Lozier, D.W.; Boisvert, R.F.; Clark, C.W. (Eds.) NIST Handbook of Mathematical Functions; Cambridge University Press: New York, NY, USA, 2010. Available online: http:/ / dlmf.nist.gov/ (accessed on 18 May 2017).

33. Sneddon, I.N. The Use of Integral Transforms; McGraw-Hill: New York, NY, USA, 1974.

34. Gradshteyn, I.S.; Ryzhik, I.M. Table of Integrals, Series, and Products, 8th ed.; Translated from Russian, Translation Edited and with a Preface by Daniel Zwillinger and Victor Moll, Revised from the 7th ed.; Elsevier/Academic Press: Amsterdam, The Netherlands, 2015. doi:10.1016/B978-0-12-384933-5.00013-8.

35. Marichev, O.I. Handbook of Integral Transforms of Higher Transcendental Functions: Theory and Algorithmic Tables; Gakhov, F.D., Ed.; Mathematics and Its Applications; Ellis Horwood Ltd.: Hemel Hempstead, UK, 1983.

36. Guo, B.-N.; Qi, F. An explicit formula for Bell numbers in terms of Stirling numbers and hypergeometric functions. Glob. J. Math. Anal. 2014, 2, 243-248. doi:10.14419/gjma.v2i4.3310.

37. Qi, F. An explicit formula for the Bell numbers in terms of the Lah and Stirling numbers. Mediterr. J. Math. 2016, 13, 2795-2800. doi:10.1007/s00009-015-0655-7.

38. Qi, F. Some inequalities for the Bell numbers. Proc. Indian Acad. Sci. Math. Sci. 2017, 127, in press. doi:10.1007/s12044-017-0355-2.

39. Qi, F.; Shi, X.-T.; Liu, F.-F. Expansions of the exponential and the logarithm of power series and applications. Arab. J. Math. 2017, 6, 95-108. doi:10.1007/s40065-017-0166-4.

40. Dana-Picard, T. Parametric integrals and Catalan numbers. Int. J. Math. Educ. Sci. Technol. 2005, 36, 410-414. doi:10.1080/00207390412331321603. 
41. Dana-Picard, T. Integral presentations of Catalan numbers. Int. J. Math. Educ. Sci. Technol. 2010, 41, 63-69. doi:10.1080/00207390902971973.

42. Dana-Picard, T. Integral presentations of Catalan numbers and Wallis formula. Int. J. Math. Educ. Sci. Technol. 2011, 42, 122-129. doi:10.1080/0020739X.2010.519792.

43. Kazarinoff, D.K. On Wallis' formula. Edinb. Math. Notes 1956, 40, 19-21.

44. Qi, F. Bounds for the ratio of two gamma functions. J. Inequal. Appl. 2010, 2010, 493058. doi:10.1155/2010/493058.

45. Qi, F. Parametric integrals, the Catalan numbers, and the beta function. Elem. Math. 2017, 72, $103-110$.

46. Dana-Picard, T.; Zeitoun, D.G. Parametric improper integrals, Wallis formula and Catalan numbers. Int. J. Math. Educ. Sci. Technol. 2012, 43, 515-520. doi:10.1080/0020739X.2011.599877.

47. Qi, F. An improper integral with a square root. Preprints 2016, 2016100089. doi:10.20944/preprints201610.0089.v1.

48. Temme, N.M. Special Functions: An Introduction to Classical Functions of Mathematical Physics; A Wiley-Interscience Publication; John Wiley \& Sons, Inc.: New York, NY, USA, 1996. doi:10.1002/9781118032572.

49. Qi, F.; Shi, X.-T.; Liu, F.-F. An Integral Representation, Complete Monotonicity, and Inequalities of the Catalan Numbers; ResearchGate Technical Report; ResearchGate Research: Berlin, Germany, 2015. doi:10.13140/RG.2.1.3754.4806.

50. Qi, F.; Shi, X.-T.; Liu, F.-F. An Exponential Representation for a Function Involving the Gamma Function and Originating from the Catalan Numbers; ResearchGate Research: Berlin, Germany, 2015. doi:10.13140/RG.2.1.1086.4486.

51. Guo, B.-N.; Qi, F. On the Wallis formula. Int. J. Anal. Appl. 2015, 8, 30-38.

52. Qi, F. Bounds for the ratio of two gamma functions: From Gautschi's and Kershaw's inequalities to complete monotonicity. Turk. J. Anal. Number Theory 2014, 2, 152-164. doi:10.12691/tjant-2-5-1.

53. Qi, F.; Li, W.-H. A logarithmically completely monotonic function involving the ratio of gamma functions. J. Appl. Anal. Comput. 2015, 5, 626-634. doi:10.11948/2015049.

54. Qi, F.; Luo, Q.-M. Bounds for the ratio of two gamma functions: From Wendel's asymptotic relation to Elezović-Giordano-Pečarić's theorem. J. Inequal. Appl. 2013, 542. doi:10.1186/1029-242X-2013-542.

55. Qi, F.; Luo, Q.-M. Bounds for the ratio of two gamma functions-From Wendel's and related inequalities to logarithmically completely monotonic functions. Banach J. Math. Anal. 2012, 6, 132-158. doi:10.15352/bjma/1342210165.

56. Qi, F.; Mahmoud, M. Some properties of a function originating from geometric probability for pairs of hyperplanes intersecting with a convex body. Math. Comput. Appl. 2016, 21, 27. doi:10.3390/mca21030027.

57. Qi, F.; Mortici, C. Some best approximation formulas and inequalities for the Wallis ratio. Appl. Math. Comput. 2015, 253, 363-368. doi:/10.1016/j.amc.2014.12.039.

58. Zhou, R.R.; Chu, W. Identities on extended Catalan numbers and their $q$-analogs. Graphs Comb. 2016, 32, 2183-2197. doi:10.1007/s00373-016-1694-y.

59. Nkwanta, A.; Tefera, A. Curious relations and identities involving the Catalan generating function and numbers. J. Integer Seq. 2013, 16, Art. 13.9.5.

60. Mitrinović, D.S.; Pečarić, J.E.; Fink, A.M. Classical and New Inequalities in Analysis; Kluwer Academic Publishers: Dordrecht, The Netherlands, 1993. doi:10.1007/978-94-017-1043-5.

61. Widder, D.V. The Laplace Transform; Princeton Mathematical Series 6; Princeton University Press: Princeton, NJ, USA, 1941.

62. Hardy, G.H.; Littlewood, J.E.; Pólya, G. Inequalities; Cambridge University Press: Cambridge, UK, 1934.

63. Marshall, A.W.; Olkin, I.; Arnold, B.C. Inequalities: Theory of Majorization and Its Applications, 2nd ed.; Springer: New York, NY, USA, 2011. doi:10.1007/978-0-387-68276-1.

64. Schilling, R.L.; Song, R.; Vondraček, Z. Bernstein Functions-Theory and Applications, 2nd ed.; de Gruyter Studies in Mathematics 37; Walter de Gruyter: Berlin, Germany, 2012. doi:10.1515/9783110269338.

65. Koshy, T.; Gao, Z.-G. Convergence of a Catalan series. Coll. Math. J. 2012, 43, $141-146$. doi:10.4169/college.math.j.43.2.141.

66. Beckwith, D.; Harbor, S. Problem 11765. Am. Math. Mon. 2014, 121, 267. doi:10.4169/amer.math.monthly.121.03.266.

67. Abel, U. Reciprocal Catalan sums: Solution to Problem 11765. Am. Math. Mon. 2016, 123, 405-406. doi:10.4169/amer.math.monthly.123.4.399. 
68. Lehmer, D.H. Interesting series involving the central binomial coefficient. Am. Math. Mon. 1985, 92, $449-457$. doi:10.2307/2322496.

69. Amdeberhan, T.; Guan, X.; Jiu, L.; Moll, V.H.; Vignat, C. A series involving Catalan numbers: Proofs and demonstrations. Elem. Math. 2016, 71, 109-121. doi:10.4171/EM/306.

70. Abramowitz, M.; Stegun, I.A. (Eds.) Handbook of Mathematical Functions with Formulas, Graphs, and Mathematical Tables; National Bureau of Standards, Applied Mathematics Series 55, 10th Printing; U.S. Government Printing Office: Washington, DC, USA, 1972.

71. Qi, F.; Guo, B.-N. Integral representations of Catalan numbers and their applications. Preprints 2017, 2017040040. doi:10.20944/preprints201704.0040.v1.

(C) 2017 by the authors. Licensee MDPI, Basel, Switzerland. This article is an open access article distributed under the terms and conditions of the Creative Commons Attribution (CC BY) license (http://creativecommons.org/licenses/by/4.0/). 